\title{
Oscillatory loads on circular cylinder with secondary structures
}

Petersen, Thor Ugelvig; Mandviwalla, Xerxes; Christensen, Erik Damgaard; Tarp-Johansen, Niels Jacob; Rüdinger, Finn

Published in:

Journal of Fluids and Structures

Link to article, DOI:

10.1016/j.jluidstructs.2020.102935

Publication date:

2020

Document Version

Peer reviewed version

Link back to DTU Orbit

Citation (APA):

Petersen, T. U., Mandviwalla, X., Christensen, E. D., Tarp-Johansen, N. J., \& Rüdinger, F. (2020). Oscillatory loads on circular cylinder with secondary structures. Journal of Fluids and Structures, 94, [102935].

https://doi.org/10.1016/j.jluidstructs.2020.102935

\section{General rights}

Copyright and moral rights for the publications made accessible in the public portal are retained by the authors and/or other copyright owners and it is a condition of accessing publications that users recognise and abide by the legal requirements associated with these rights.

- Users may download and print one copy of any publication from the public portal for the purpose of private study or research.

- You may not further distribute the material or use it for any profit-making activity or commercial gain

- You may freely distribute the URL identifying the publication in the public portal 


\section{Oscillatory loads on circular cylinder}

2 with secondary structures.

4 Thor Ugelvig Petersen ${ }^{1}$, Xerxes Mandviwalla ${ }^{1}$, Erik Damgaard Christensen ${ }^{2}$, Niels

5 Jacob Tarp-Johansen ${ }^{3}$, and Finn Rüdinger ${ }^{3}$

1) DHI, Port and Offshore Department, Agern Alle’, DK-2970 Hørsholm, Denmark.

\section{Abstract}

2) Technical University of Denmark, DTU-MEK, Nils Koppels Alle', Building 403, DK-2800 Kgs. Lyngby, Denmark

3) Ørsted, Wind Power, Nesa Allé 1, DK-2820 Gentofte, Gentofte.

This study deals with a numerical and experimental study of the secondary structures effect on the load on the main circular cylinder. Its objective is to address the increasing amount of offshore structures installed as part of offshore wind farms. The typical support structure for a wind turbine is a monopile foundation. Boat-landings are attached to the monopile and these structures act as secondary structures. Forces on a cylinder with and without secondary structures attached in unsteady flows have been measured experimentally and simulated numerically. The results of the tests were compared to each other and to existing work in the present report for validation of the CFD model. The secondary structures were represented by circular cylinders placed at different orientations around the main circular cylinder. All the analyses assumed uniform conditions transverse to the flow direction (i.e. 2D set-up). The numerical simulations was based on the OpenFOAM CFD tools. The validation tests with a circular cylinder without secondary structures showed good agreement between the physical and numerical results, as well as to the results available in the open literature. The orientation of the secondary structures had a clear influence on the drag related part of the force as the drag coefficient $C_{D}$ when the secondary structure was orientated towards $\theta=90^{\circ}$, i.e. perpendicular to the flow direction. The inertia part of the force, i.e. the inertia force coefficient $C_{M}$, was only affected by the orientation to small extend. 


\section{Introduction}

32 For the last couple of decades European oceans have been subject to massive

33 development of offshore wind farms. The development is foreseen to be supplemented

34 by a more general implementation of marine infrastructure in the near future, see.

35 (Christensen et al., 2015). The development includes energy facilities, e.g. offshore wind farms, exploitation of wave energy, and development and implementation of marine aquaculture.

Support structures for offshore wind farms are often monopiles. Other support structures used have been jacket and gravity based structures. Boat-landings are attached to the support structure, e.g. a monopile, and these structures act as secondary structures. Several studies have investigated the total load on wind turbine support structures. (Christensen et al., 2005) studied wave forces and wave run-up on a vertical, cylinder in non-breaking and breaking waves with computational fluid dynamics (CFD). (Bredmose et al., 2006) studied the wave loads on a gravity based foundation with CFD and compared this with experimental results. (Christensen et al., 2011) studied irregular breaking wave loads on a gravity based structure. (Paulsen et al., 2014) studied the forces from steep waves on a vertical cylinder with CFD. Several other studies have also studied the forces on the large main structure. The literature is weak on the studies of forces on secondary structures, but some literature exists on wave forces on for instance a service platform in (Damsgaard et al., 2007) and (Bredmose and Jacobsen, 2011). Studies on and the effect on the main structure of other secondary structures, such as boat-landings, are scarce. However, the boat-landings are an important structural element as they provide access to the offshore wind turbine from the seaside. In the following we address some fundamental studies that is related to the analyses presented in this paper.

The forces on a circular cylinder have been intensively investigated. A general introduction to hydrodynamics around and forces on cylindrical structures are given in (Sumer and Fredsøe, 2006). (Sarpkaya, 1976), and (Sarpkaya, 1986) experimentally analysed the forces on a circular cylinder in oscillatory flow generated in a u-tube. The experiments that included smooth as well as sand-roughed cylinder surfaces showed

61 some general trends; a minima of the inertia coefficient $C_{M}$ and a maxima of the drag coefficient $C_{D}$ for the Keulegan-Carpenter number $(K C)$ around 10 -15. Increasing 
roughness results in larger drag coefficients and smaller inertia coefficients for higher Reynolds numbers. (Sarpkaya, 1977) extended the analyses to include a circular cylinder near a wall. Other examples of research on oscillatory loads on a circular cylinder are (Bearman et al., 1986) who studied theoretical and experimentally the oscillatory load on a circular cylinder, (Bearman et al., 1984) set up a model equation for the transverse forces in oscillatory flow, (Justesen, 1989) studied oscillatory loads using an oscillating carriage instead of an oscillating fluid and (Justesen, 1991) carried out a numerical study of the flow around a cylinder in oscillatory flow that included estimation of force coefficients. These studies are in line with the studies of (Sarpkaya, 1976), and (Sarpkaya, 1986), and are extensions and supplementary investigations of oscillatory loads with different experimental set-up, numerical methodologies, and extension of the parameter space.

There exists other studies that examined the forces on different configurations on smaller and larger cylinders. In connection with pipelines an often used layout is the piggy back layout of a smaller pipe line on top of a larger pipeline. For instance a piggy-back line system comprise a primary line employed for export of the hydrocarbon flow away from a field with a secondary smaller diameter flow line carrying injection water, injection chemicals etc. to the field. (Kalghatgi and Sayer, 1997) studied forces on piggy-back configurations in steady incident flows. (Cheng et al., 2012c) studied the forces on a large and small cylinder in piggy-back position near a seabed experimentally. Experiments were carried out in a wave flume and both regular waves as well as irregular waves were used in the investigations. In all cases the small cylinder was on top of the large cylinder. (Zhao et al., 2007) used numerical modelling to study the forces on a piggy-back system in steady current. (Cheng et al., 2012a) and (Cheng et al., 2012b) studied the wave forces on a piggy-back system, where they used a VOFmethod (Volume of Fluid) to track the free surface.

Several authors have studied tandem arrangements of two circular cylinders, such as (Alam et al., 2005). Other studies of open structures is for instance jacket structures modelled as a porous medium, (Santo et al., 2018a), (Santo et al., 2018b), and fish cages (Chen and Christensen, 2016).

In this study we focused on modelling the secondary structures directly in the 94 experimental and numerical modelling. The main objective of our study was to assess 
95 the effect of the secondary structures on the total load on the support structure. The

96 motivation behind the study was to determine with improved accuracy the forces from wave

97 interaction with the wind turbine foundations and the secondary structures. The future use is

98 expected to be exploited with engineering tools that estimates forces with the Morison force

99 model rather than a direct simulation of the forces in CFD. The methodology was based on

100 a combination of physical experiments supplemented with CFD-calculations. The CFD-

101 calculations should support the findings of the physical experiments and to clarify some

102 uncertainties of the experiments. This involved assessing the possible scale effects.

103 The outline of the paper is as follows. First we describe the experimental set-up in

104 section 2. This includes the set-up of the flume, carriage and the physical models that

105 were tested, under different oscillating flow condition. The section also describes how

106 basic analyses were carried out of for instance the method to assess the force

107 coefficients that was the same in the physical experiment and the numerical

108 calculations. The section on the experiments is followed with section 3 that describes

109 the numerical method that was based on the OpenFOAM CFD library, ("OpenFOAM,

110 The open source CFD toolbox," 2018). In this section special attention is given to the

111 description of the oscillating body force that modelled the effect of an oscillating flow.

112 Section 4 presents a comparison between the experimental and numerical results to

113 well-known reference works in the literature for a single circular cylinder. Section 5

114 presents the results for a cylinder with secondary structures. The focus was on the

115 orientation of the secondary structure and the influence of the KC-number. Finally,

116 section 6 summarises and concludes the work. 


\section{Experimental set-up and test conditions}

119 The experiments were carried out in the towing tank at the Technical University 120 of Denmark (DTU). The measurement section of the tank was 35m long, 3.0m wide and 121 1.0m high. A carriage running on rails on top of the flume can operate at up to about $1221.5 \mathrm{~m} / \mathrm{s}$ back and forth in an otherwise calm water. The flume is sketched in Figure 1.

123

124

125

126

127

128

129

130

131

132

\section{3}

134

135

136

137

138

139

140
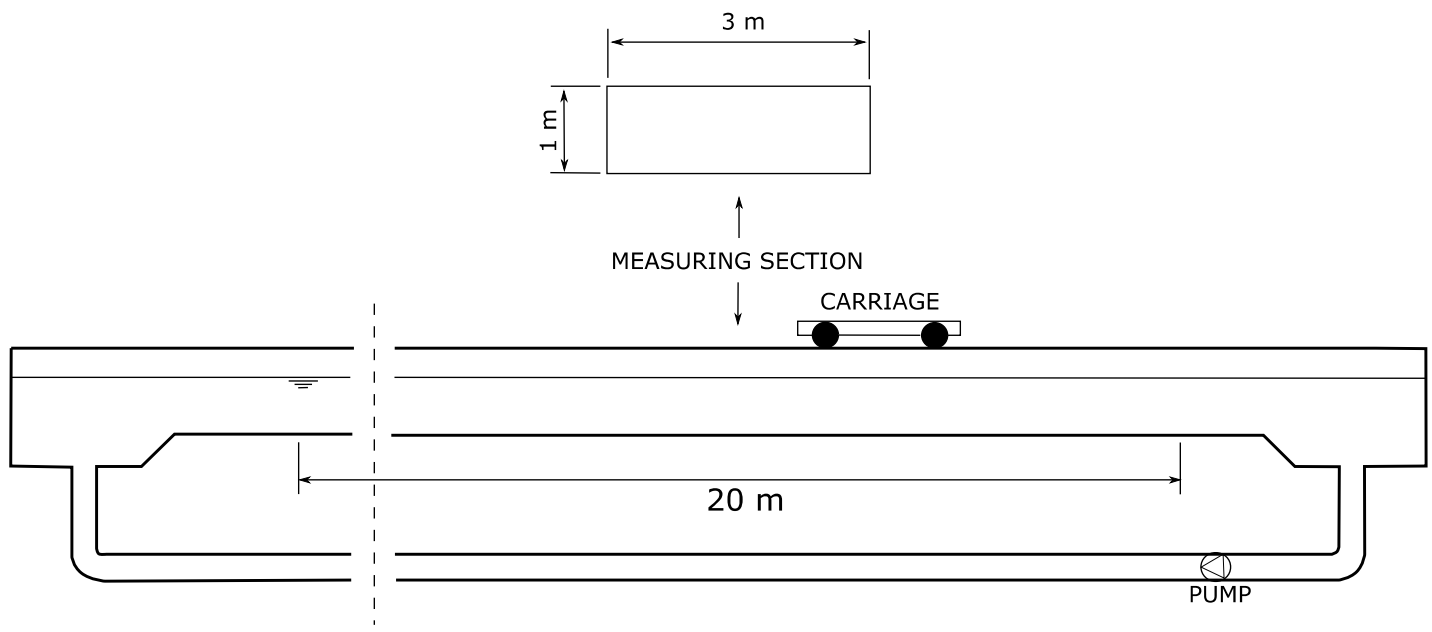

Figure 1 Schematic description of the test flume.

The relatively large size of the experimental facility was important as the side walls of the experimental facility induce a confining effect on the model, which normally is termed blockage. Generally, one may assume that the blockage effect is small and may be ignored when the ratio of the cylinder diameter to the width of the experimental facility, $\mathrm{D} / \mathrm{B}$, is less than 0.1 . In the present situation, blockage effects were undesirable and the value was maintained so $\mathrm{D} / \mathrm{B} \leq 0.1$.

\subsection{Test conditions}

The study of the effect of secondary structures on the hydrodynamic forces was related to two common designs in offshore wind engineering. A very common support structure is the monopile, which also include as boat-landing for assessing the wind turbine for inspection purposes. Another common support structure is the jacket structure. A jacket structures can be used for support structures for wind turbines, but is also often used for supporting facilities such as transformer stations, and accommodation. The boat-landing is in these cases typically attached to just one of the 
141 jacket legs. On this background two typical two-dimensional cross-sections for support

142 structures with secondary structures were established.

143 The monopile with secondary structures consisted of a monopile with a diameter

144 of 6.0 metres, and four smaller circular cylinders, where the two smallest had a diameter

145 of $0.177 \mathrm{~m}$ and the remaining two a diameter of $0.419 \mathrm{~m}$. The smaller diameter

146 cylinders represent ladders and the larger boat landings. The jacket leg had a diameter

147 of $1.80 \mathrm{~m}$, and the secondary structure were represented by 6 smaller cylinders, where

148 three had the diameter $0.146 \mathrm{~m}$ and the other three had a diameter of $0.386 \mathrm{~m}$. All

149 measures relate to full scale. Figure 2 shows a sketch of the two cross-sections. For the

150 monopile the total cross-sectional area, including the secondary structures, was $A_{\text {tot }}=$

$15128.6 \mathrm{~m}^{2}$, which was approximately $1 \%$ more the main structure. For the jacket structure

152 the total area of the cross section was $A_{t o t}=2.935 \mathrm{~m}^{2}$, which was approximately $15 \%$ of

153 the main jacket leg alone.

154
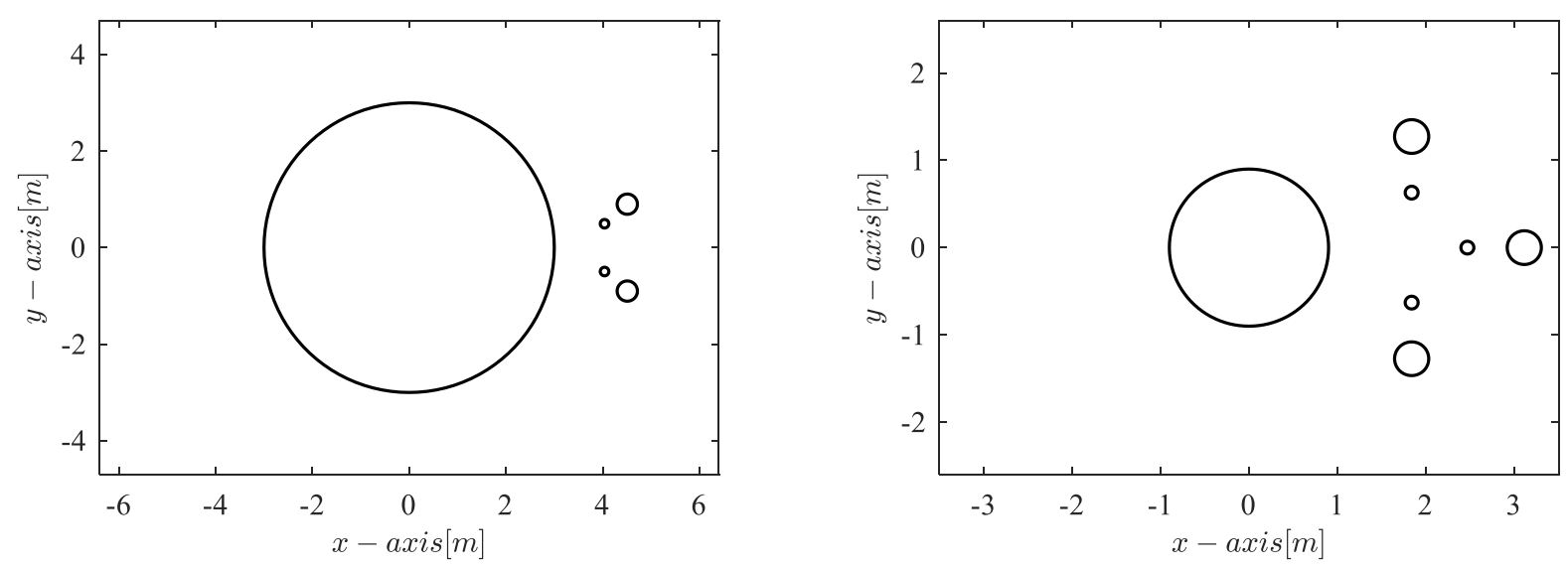

155

Figure 2 The two layouts, Left: Monopile with secondary structures, Right: Jacket leg

156 with secondary structures.

157 
Table $1 \quad$ Full scale data used in the tests. The data reflect typical dimensions on wind turbine support structures.

\begin{tabular}{|l|l|l|l|}
\hline Property & $\begin{array}{l}\text { Variable name } \\
\text { / Unit }\end{array}$ & Monopile & Jacket leg \\
\hline & & Full scale & Full scale \\
\hline Diameter & $\mathrm{D}_{1}(\mathrm{~m})$ & 6 & 1.8 \\
\hline Boat landing & $\mathrm{D}_{2}(\mathrm{~m})$ & 0.419 & 0.386 \\
\hline Ladder & $\mathrm{D}_{3}(\mathrm{~m})$ & 0.177 & 0.146 \\
\hline Wave period & $\mathrm{T}(\mathrm{s})$ & 15 & 15 \\
\hline Amplitude & $\mathrm{a}(\mathrm{m})$ & $12.5,10,7.5,5.0,2.5$ & $12.5,10,7.5,5.0,2.5$ \\
\hline $\begin{array}{l}\text { Angular } \\
\text { frequency }\end{array}$ & $\omega=2 \pi / T(\mathrm{rad} / \mathrm{s})$ & 0.42 & 0.42 \\
\hline
\end{tabular}

160

The flow around the monopile and the jacket leg lies within the classic topic of hydrodynamics; flow around a circular cylinder in waves. The flow around a circular cylinder in waves exerts a resultant in-line force (drag and inertia) and cross-flow force (lift) on the cylinder; a thorough account on the topic is provided in (Justesen, 1991). Cylinder diameter and surface roughness both influence the resultant force as well as the velocity, period and amplitude of the wave motion. Generally, the effect of cylinder diameter, $D$, maximum horizontal velocity, $U_{m}$, and the amplitude of the wave motion, $a$, is expressed in terms of two non-dimensional numbers: the Reynolds number, $\operatorname{Re}=D U_{m} / v$, and the Keulegan-Carpenter number, $K C=U_{m} T / D$. Surface roughness is expressed in terms of the relative roughness, $k_{s} / \mathrm{D}$, where $\mathrm{k}_{\mathrm{s}}$ is the equivalent sand roughness (or $k / D$ where $k$ is the roughness height).

A vertical cylinder with secondary structures under exposure to waves experiences alterations in the drag and the lift force compared to a single plain cylinder. These effects are attributed to:

176 1. The deviation in the deflecting flow lines around the cylinder with the secondary

177 structures attached

178 2. The change of the plan view separation point of the lee-wake vortices 
3. The increase in the hydrodynamic field enforced by the secondary cylinders. Obviously, the plan view orientation, $\theta$ as shown in Figure 3 of the secondary structures relative to the KC-number and the Reynolds number will be the governing parameters.

Due to mutually exclusive scaling laws, dynamic similarity between physical model experiments and full scale is only attainable at full scale. The physical model experiments were scaled using Froude scaling. Froude scaling together with geometrical similarity allowed reproduction of the Keulegan-Carpenter number, $\mathrm{KC}$, orientation of the secondary structure, $\theta$, and the relative roughness, $\mathrm{k}_{\mathrm{s}} / \mathrm{D}$, in the model. The orientation of the secondary structure, $\theta$, is defined in Figure 3Fejl!

Henvisningskilde ikke fundet.. This, however, meant that the Reynolds number was not scaled correctly in the physical model experiments. The consequences of this, however, were limited because of the surface roughness and the application of tripwires inside the developing boundary layer around the cylinder, which will be detailed later.

Oscillating motion
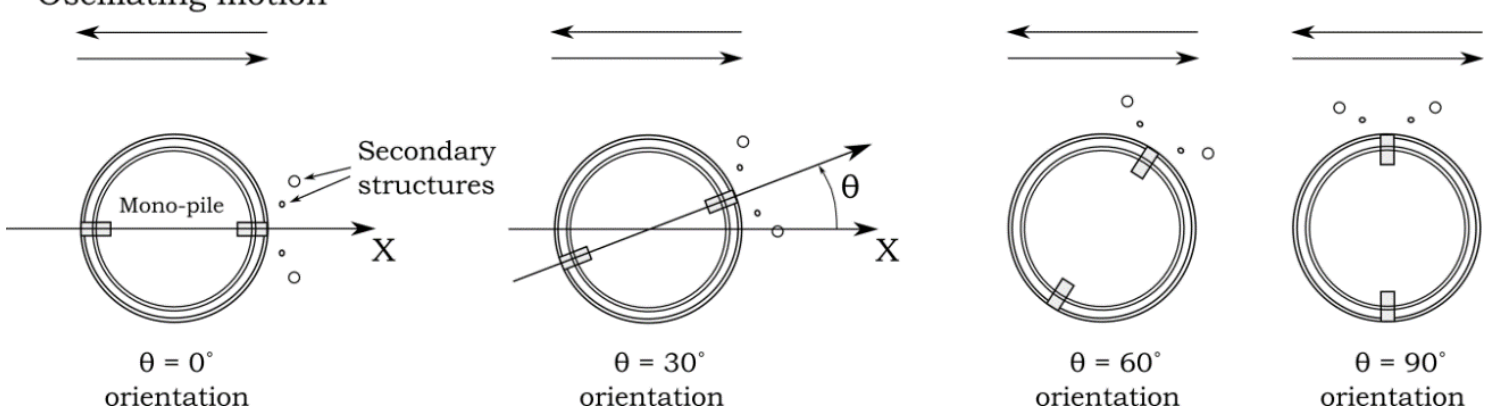

Figure 3 Definition sketch (schematic) of the monopile set-up with the orientation of the secondary structures relative to the direction of oscillatory motion defined by $\theta$.

\subsubsection{Model scale}

The physical model experiments were scaled according to Froude number scaling:

$$
\begin{aligned}
F r_{\text {full-scale }} & =F r_{\text {model }} \\
\left(\frac{u}{\sqrt{g h}}\right)_{\text {full-scale }} & =\left(\frac{u}{\sqrt{g h}}\right)_{\text {model }}
\end{aligned}
$$

in which $\mathrm{u}$ is the velocity and $\mathrm{h}$ the water depth. Table 2 gives factors for scaling of full scale to model scale in the monopile and the jacket leg set-up. The monopile structure was tested at a scale of 1:21.4 and the jacket leg at scale 1: 12.9. 
Table 2 Factors for scaling of full scale to model scale. Monopile with $\lambda=21.4$ and 207 jacket leg with $\lambda=12.9$

208

\begin{tabular}{|c|c|c|c|}
\hline Parameter & Scale law & $\begin{array}{c}\text { Scale factor for } \\
\text { monopile }(\boldsymbol{\lambda}= \\
\mathbf{2 1 . 4})\end{array}$ & $\begin{array}{c}\text { Scale factor } \\
\text { jacket leg }(\boldsymbol{\lambda}= \\
\mathbf{1 2 . 9})\end{array}$ \\
\hline Length & $\lambda$ & 21.4 & 12.9 \\
\hline Area & $\lambda^{2}$ & 458 & 166 \\
\hline Volume & $\lambda^{3}$ & 9800 & 2147 \\
\hline Time & $\lambda^{1 / 2}$ & 4.62 & 3.59 \\
\hline Velocity & $\lambda^{1 / 2}$ & 4.62 & 3.59 \\
\hline Mass & $\lambda^{3}$ & 9800 & 2147 \\
\hline Force & $\lambda^{3}$ & 9800 & 2147 \\
\hline Moment & $\lambda^{4}$ & 209,727 & 27,692 \\
\hline Pressure & $\lambda$ & 21.4 & 12.9 \\
\hline
\end{tabular}

209

210 The diameter and length of the measurement sections for the monopile and the 211 jacket leg were:

Monopile: $\quad D_{\text {model }}=0.280 \mathrm{~m}, L=0.300 \mathrm{~m}$

$$
\text { Jacket leg: } \quad D_{\text {model }}=0.140 \mathrm{~m}, L=0.300 \mathrm{~m}
$$

214 2.2.2 Submerged cylinder with and without secondary structures

Figure 4 shows the submerged circular cylinder with and without secondary

216 structures attached. The cylinder was suspended from an adjustable support frame,

217 which allowed the vertical position of the cylinder in the water column to be adjusted.

218 A close-up of the support frame is shown in Figure 5. The support frame consisted of

219 two box profiles (placed on top of the carriage), four M20 threaded steel rods and two

$220200 \mathrm{~mm} \times 400 \mathrm{~mm} \times 15 \mathrm{~mm}$ steel plates assembled with nuts and washers. The set-up of

221 the support frame, cantilever block and the connection to the steel liner, shown in

222 Figure 5, enabled rotation of the model relative to wave direction by simply rotating the 223 steel liner around the cantilever block. 

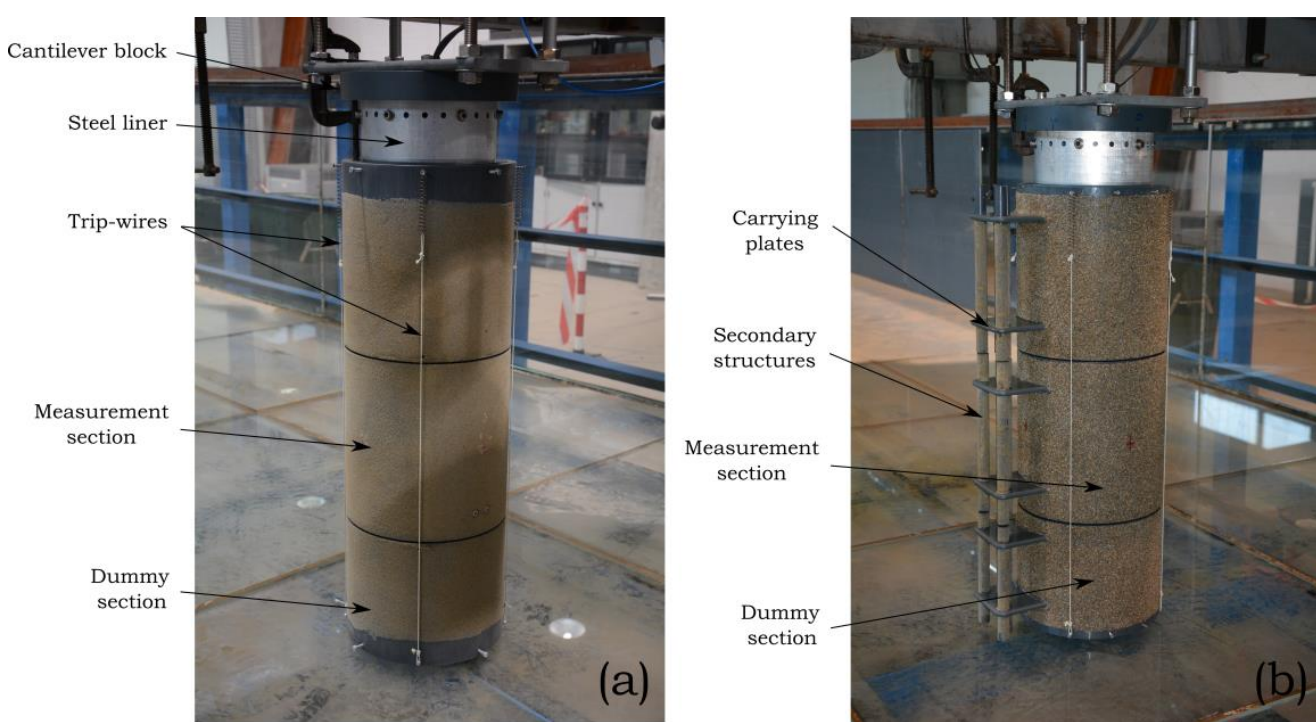

Figure 4 (a) Monopile without secondary structures (b) Monopile with secondary structures.
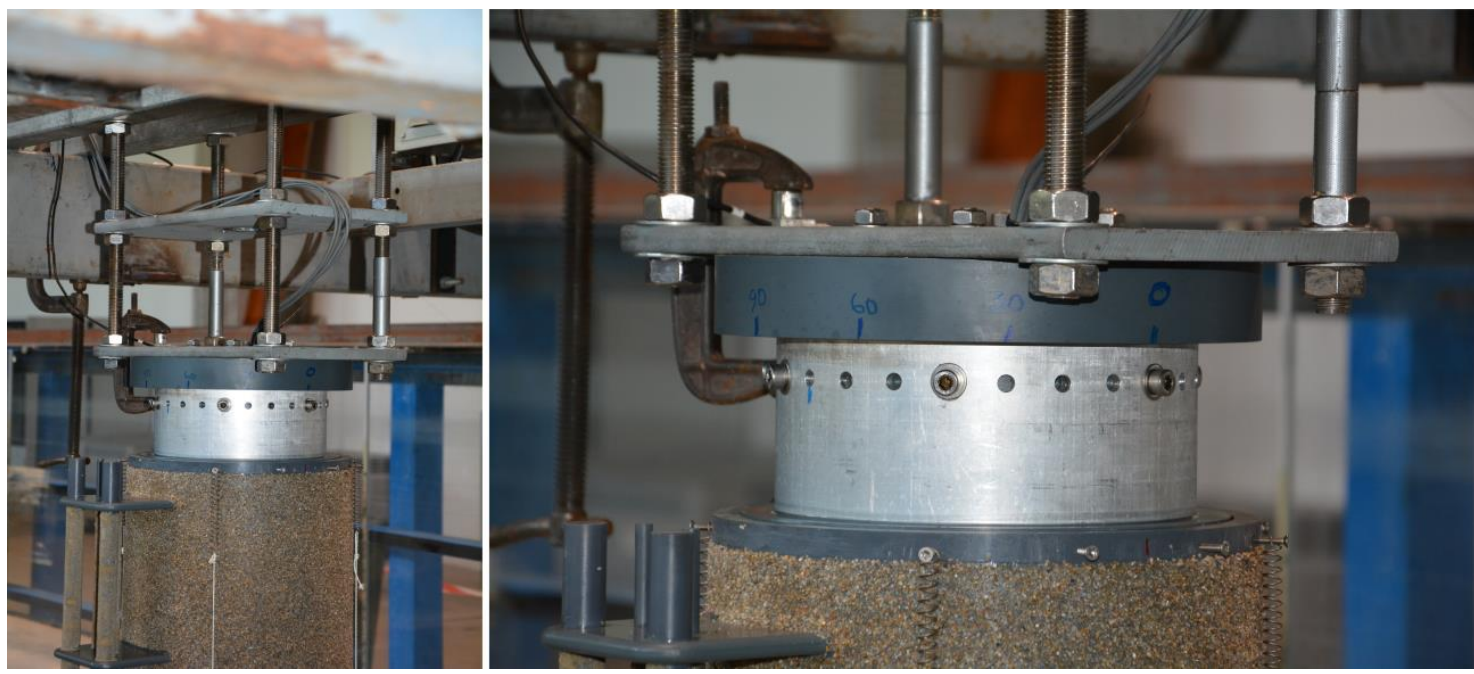

Figure 5 Left) Support frame cantilevering the monopile model. (Right) Close-up of connection between support frame and the suspended monopile model

The models were split into three parts Figure 4. The middle part was the measurement section, while the bottom and top parts were dummy sections. The dummy sections were rigidly fixed to the inner steel liner, while the measuring section was suspended in two (2-component) force gauges capable of measuring the two horizontal forces, in-line and lift $\left(F_{x}, F_{y}\right)$. The force gauges were attached asymmetrically to the inside of the steel liner. One force gauge at the top (between dummy and measuring sections) and one force gauge at the bottom of the measuring section. One end of each of the two force gauges was rigidly fixed to the steel liner, while the other end of the force gauges held the measurement section. A small gap 
between the outer PVC cylinder and the inner steel liner allowed for slight movement of the measuring section relative to the steel liner so that the displacement could be measured by the strain gauges on the force gauges.

244 The measurement section was mounted on two 2-component force gauges. The two force gauges of type DHI-205/150 (jacket leg set-up) and DHI-205/150 (and DHI205/100) (monopile set-up), were calibrated independently and checked after assembly of the model. They were connected to strain amplifiers of type DHI-106E. Figure 6

248 illustrates the load cells attachment to the physical model.
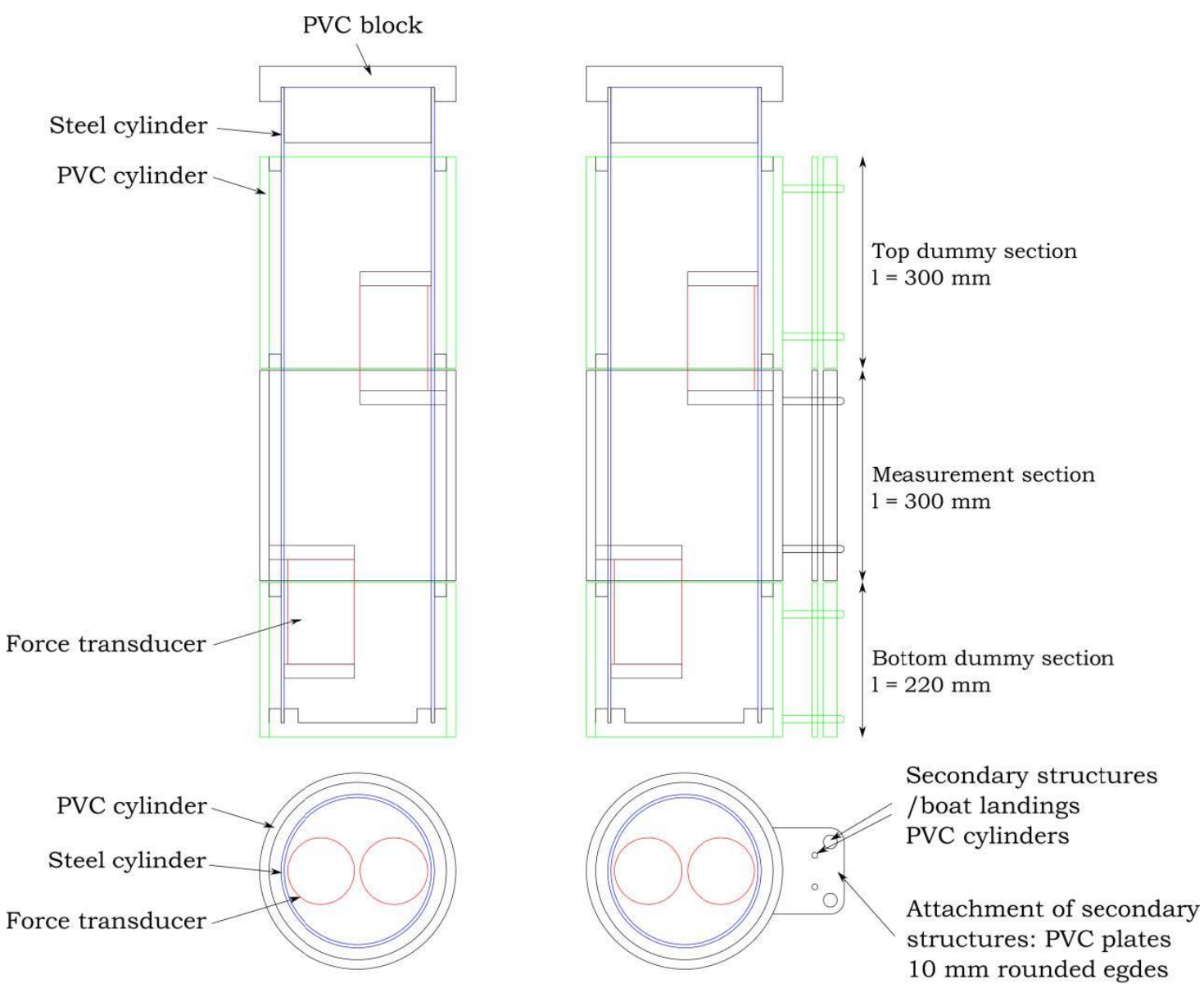

Figure 6 Sketch of arrangement of the load cells.

$10 \mathrm{~mm}$ rounded egdes

Figure 7 sketches how the physical model attached to the carriage. The flow around an object placed in a channel with a free surface induces a local set-up and set-down of the water table due to the pressure variations around the model. These disturbances are increased fast with increasing velocities. There seems to be two effective ways of damping the effect of this set-down: (1) to place a plate/screen below the surface shielding the model against the set-down (i.e. the screen shall be 

section sufficiently low beneath the set-down level for the free surface effects not to interfere with the flow around the measurement section.

In the present case, the second option was chosen, where the top of the measurement section was below 0.23 m below the free water surface. The maximum set-down effects were observed to penetrate the free surface water level down to about $7 \mathrm{~cm}$.

Besides the direct surface deformation, a wave field is induced to the rear/downstream of the model with the same characteristics as the wave patterns around a ship hull. The wave field can give rise to additional forces on the models, but due to the rather short wavelengths, the force is concentrated at and just below the surface.

The effects of surface deformations and wake waves are in spite of their magnitude in the high-speed tests considered low in the present experiments mainly due to the distance from the free surface level to the top of the measurement section.

Figure 7 Illustration of the model attached to the carriage. The measuring section was $30 \mathrm{~cm}$ high. The total water depth was $0.75 \mathrm{~m}$. The top of the measuring section was $0.23 \mathrm{~m}$ below the free water surface.

\subsubsection{Trip wires}

280 For the selected range of the model tests, the Re numbers were in the critical,

281 super-critical or post-critical range. To ensure the transition from a laminar to a

282 turbulent boundary layer around the main cylinder, trip wires were applied, as

283 previously mentioned and visible in Figure 4. The trip wires ensured an external 
generation of turbulence in the developing boundary layer around the surface of the main cylinder, and in turn this should render a measured force response similar to the post-critical range of the Re-number, even though at model scale the Re-number corresponded to the critical or super-critical range. The use of trip wires is a well known technique, see for instance (James and Truong, 1972), (Pearcey et al., 1982), (Igarashi, 1986) and (Hover et al., 2001).

The trip wires were placed at a plan view angle $\theta= \pm 45^{\circ}$ to the oscillating direction (x-direction) on both sides of the main cylinder. This plan view position of the trip wires was maintained as the cylinder with the secondary structures was reorientated. No trip wires were applied in the case where the location of the secondary structures was in conflict with placing the trip wires (i.e. directly on top of each other); in these cases the added hydrodynamic field enforced by the secondary structures was assumed to make up for the missing trip wires. The trip wires applied to the models had a diameter of $1.6 \mathrm{~mm}$ and were located with a plan view radial distance of $2-3 \mathrm{~mm}$ from the surface of the main cylinder for the most part of the test programme, while some specific tests were run with smaller diameter trip wires or the trip wires were removed.

300 In both cases, the purpose was to measure the effect of the trip wires on the vortex shedding and in turn on the recorded in-line forces.

The CFD calculations used a full-scale set-up. This include fully rough boundary conditions for the for instance the turbulence model. Therefore, no trip wires were used in the CFD model that is described in detail later. In this way the CFD model tests the assumption of the use of trip wires. Using a combination of physical model tests at model scale and CFD modelling at full scale has previous been used to study complex flow phenomenon. See for instance (Jensen et al., 2018).

\subsubsection{Data acquisition}

310 Data acquisition was performed using the DHI Wave Synthesizer (WS) data

311 acquisition system. The measurements were logged and data stored at a sampling 312 frequency of $160 \mathrm{~Hz}$ at model scale. The measurements contained the time series of 313 force records $\left(F_{x}, F_{y}\right)$, displacement and velocity of carriage. The carriage position, x, 314 along the length of the flume was measured with a SICK DT50 (P2113) laser distance 315 measuring device. The measurement range was $0.2 \ldots 20 \mathrm{~m}$, with a resolution less than 1 
$316 \mathrm{~mm}$, and with an accuracy of $\pm 1 \mathrm{~mm}$ with our use. For longer distances and less

317 remission the accuracy is less, e.g. $\pm 7 \mathrm{~mm}$. The post-processing will typically narrow the

318 accuracy further as the accuracy above refers to the instantaneous accuracy. During

319 post-processing, the position signal was low-pass filtered with a cut-off frequency of 1

$320 \mathrm{~Hz}$ and differentiated with time to give the velocity of the carriage, $u=d x / d t$, and the

321 acceleration $d u / d t$.

322 The subsequent analysis of the time series of load measurements was post-

323 processed and analysed through the following procedure:

324 - The time series of load measurements were filtered by a once-through band pass

325 filter removing network noise at a low pass filter level of $10 \mathrm{~Hz}$. This is similar to

326 previous research that used the carriage system, e.g. (Jensen et al., 2018), (Bryndum

327 et al., 1992).

328

- The loads records of the individual wave periods of oscillations were assembled to give the mean force record, mean velocity and acceleration over the period of oscillatory motion.

\subsubsection{Accuracy of the tests}

The uncertainty of the physical model test was assessed where the effects of free surface, blockage effect, secondary motions from for instance horse shoe vortices', correlation length of vortex shedding, and workmanship of the physical model were considered. Some of these quantities have also been discussed elsewhere. All of the above-mentioned phenomena contribute to an inaccuracy of the test results. The below list gives possible sources of inaccuracies with a best estimate of their magnitude:

1. Workmanship of model $1 \%$

2. Blockage effects $0.5 \%$

3. The force measuring system $0.5 \%$

4. Free surface effects low 
The summation of the inaccuracies listed above to a total inaccuracy for the experimental results of the force measurements are estimated based on previous experience with similar model set-ups to be 5-8\%. Note that this estimate does not consider the correlation length and the lacking Re number scaling.

\subsection{Data analyses}

For a cylinder accelerated and moved in still water, the Morison formulation for the total in-line force reads:

$$
F_{\text {inline }}=1 / 2 \rho C_{D} D h U|U|+\rho C_{M} A h \frac{d U}{d t}
$$

The first term on the right-hand side is the drag force and the second term is the hydrodynamic mass force (inertia force), $\rho$ is the water density, $h$ is the cylinder height, $D$ is the cylinder diameter, $A$ is the cross-sectional area, $U$ is the undisturbed velocity relative to the centre line of the position of structure but without structure in place. The velocity $U$ could in our case is also the velocity far away from the structure relative to the position of the structure. $C_{D}$ is the drag coefficient and $C_{M}$ is the hydrodynamic mass coefficient.

The drag coefficient, $C_{D}$, and the hydrodynamic mass coefficient, $C_{M}$, were determined for each of the experimental and numerical tests. The technique used for determining the force coefficients was the method of least squares fit, see for instance (Sumer and Fredsøe, 2006). In all cases the diameter and cross-sectional area of the main structure was used. For instance for the monopile with secondary structures $D=$ $6.0 \mathrm{~m}$ and $A=28.27 \mathrm{~m}^{2}$ eqs. (2). The total frontal area increases as the position of the secondary structure is turned perpendicular to the flow direction. This effect was not accounted for by adjusting the diameter and cross sectional area in the analyses. Instead the effect will be seen in modified force coefficients.

The measurement section consisted of a cylinder of PVC, fixation units of

373 aluminium, force gauges and screw bolts as mentioned previously. Further to this, the compartments within the model (between PVC shell and steel liner; and inside steel liner) were filled with water during testing. Therefore, the measured inertia force

376 included a contribution from the entire mass of the measurement system and the water 377 inside. 

section and the water inside is described in the following. Firstly, the assembled models were tested in the flume without water. Here the model was oscillated to achieve $K C \approx$ 1 , and the measured maximum force response comprised only the accelerated mass of the measurement section. It should be mentioned that there was a good agreement between the measured force response and the calculated force response in this procedure. Secondly, the model was submerged in still water and oscillated at the same test conditions (i.e. $K C \approx 1$ ) where $C_{m}=1$. Hence, the measured inertia force could be described by:

$$
\begin{aligned}
& \rho C_{m}^{\prime} A h \frac{d U}{d t}=\rho C_{m} A h \frac{d U}{d t}+\rho n A h \frac{d U}{d t} \\
& C_{m}^{\prime}=C_{m}+n
\end{aligned}
$$

where the last term, $n$, on the right-hand side represents the mass of the measurement section and the water inside (not to be confused with the Froude-Krylov force). The hydrodynamic force coefficient, $C_{m}$ was therefore determined from the actual measured inertia coefficient, $C_{m^{\prime}}$, using the above relationship: $C_{m}=C_{m}^{\prime}-n=1$ at the given flow conditions $(K C \approx 1)$. $n$ remained constant for a specific model setup with changing wave flow climates (with specific weight of model and water inside), and this procedure was remade for each model setup tested in the present experimental campaign. In the present cases, $\mathrm{n}$ assumed values $O(0.9-1.1)$ and subtraction was made in the measured force time series accordingly.

Lastly, to obtain the inertia coefficient $C_{M}$ :

$$
C_{M}=C_{m}+1
$$

400 pile geometry, and therefore the effect of the secondary cylinders were inherent in the

401 force coefficients, drag and added mass, obtained through the present analysis. For the 402 practical application of the available force coefficients one should re-evaluate the 403 Froude-Krylov effect of the secondary structures relative to the main pile geometry. 404 In the case with secondary structures to the main cylinder, the mass of the 405 secondary structures times the acceleration were also removed from the measured force 406 response. 

force (or lift force in the following) was addressed in the analyses of the single circular cylinder for comparison with literature. The maximum value of the lift force was considered for both the physical and CFD models.

$$
F_{L, \max }=\frac{1}{2} \rho C_{L, \max } D h U_{\mathrm{m}}^{2}
$$

\section{Numerical Methodology}

The model meshing and numerical calculations were performed using the $\mathrm{C}++$ CFD toolbox OpenFOAM® (OF) (“OpenFOAM, The open source CFD toolbox,"

416 2018). OF is open source and freely distributed, thus allowing and enabling researchers

417 to modify the standard code to suit the needs of a specific project or task.

\subsection{The numerical model}

Stokes equations (URANS) with the body force term:

$$
\begin{gathered}
\frac{\partial \bar{u}_{i}}{\partial x_{i}}=0 \\
\frac{\partial \bar{u}_{i}}{\partial t}+\bar{u}_{j} \frac{\partial \bar{u}_{i}}{\partial x_{j}}=-\frac{1}{\rho} \frac{\partial p}{\partial x_{i}}+\frac{\partial}{\partial x_{j}}\left[v\left(\frac{\partial \bar{u}_{i}}{\partial x_{j}}+\frac{\partial \bar{u}_{j}}{\partial x_{i}}\right)-\overline{u_{i}^{\prime} u_{j}^{\prime}}\right]+F_{B i}
\end{gathered}
$$

424 where $\bar{u}_{\mathrm{i}}$, are the time-averaged velocities (phase-averaged for oscillatory flow), $p$ is the pressure, $v$ is the kinematic viscosity of water, $\rho$ is the density of water, $x_{i}$ are Cartesian coordinates and $\mathrm{t}$ is time.

The body force term in eqs. (8) to model the oscillating wave is denoted by $\mathrm{F}_{\mathrm{Bi}}$ and is set according to eqs. (8) below:

$$
\begin{aligned}
& F_{B_{1}}=\omega U_{m} \cos (\omega t+\varphi) \\
& F_{B_{2}}=0
\end{aligned}
$$

where $\omega$ is the angular wave frequency, $U_{m}$ is the maximum wave velocity in the free stream, and $\varphi$ is a phase shift. $F_{B_{1}}$ and $F_{B_{2}}$ are the components of the body force in the $x$ and $y$ directions, respectively. 
Alternatively to body force terms you could specify the flow velocity at the inlet.

435

436

437

438

439

440

441

442

443

444

445

446

447

448

450

451

452

453

456

457

458

This would create a pressure gradient as for instance under waves. This pressure gradient will influence on the cylinder as the Froude-Krylov force does under waves. We used the body force term and therefor did not include a pressure gradient from the ambient water. The inertia coefficient had to be compensated for that when the results is used in waves, just as we have to compensate the inertia coefficients estimated from our physical model tests with a moving carriage.

Model scenarios, where the angle of the incident was tested, were modelled by rotating the mesh geometry as presented in the next section. The body force remained fixed in the horizontal direction.

The Reynolds stress tensor term, $-\overline{u_{i}^{\prime} u_{j}^{\prime}}$, in (7) is equated to the Boussinesq eddy viscosity assumption as follows in eqs. (9):

$$
\overline{u_{i}^{\prime} u_{j}^{\prime}}=v_{T}\left(\frac{\partial \bar{u}_{i}}{\partial x_{j}}+\frac{\partial \bar{u}_{j}}{\partial x_{i}}\right)-\frac{2}{3} k_{T} \delta_{i j}
$$

where $v_{T}$ is the turbulent eddy viscosity, $k_{T}$ is the turbulent kinetic energy and $\delta_{i j}$ is the Kronecker delta.

In order to close the system of equations, the eddy viscosity term was modelled by solving the two-equation k-Omega SST (Shear Stress transport) turbulence model. This turbulence model was selected for its merit in modelling flows where adverse pressure gradients and flow separation are important. It is a blended model which utilises the strength of the k-epsilon model in the outer regions of free shear flow and the k-omega model in the near wall regions (Menter, 1994).

$$
\begin{aligned}
& \rho \frac{\partial k}{\partial t}+\frac{\partial \rho k \bar{u}_{i}}{\partial x_{j}}=\frac{\partial}{\partial x_{j}}\left[\left(\mu+\mu_{t} / \sigma_{k}\right) \frac{\partial k}{\partial x_{j}}\right]+\tau_{i j} \frac{\partial \bar{u}_{i}}{\partial x_{j}}-\rho \beta^{*} k \omega \\
& \rho \frac{\partial \omega}{\partial t}+\frac{\partial \rho \omega \bar{u}_{i}}{\partial x_{j}}= \frac{\partial}{\partial x_{j}}\left[\left(\mu+\mu_{t} / \sigma_{\omega}\right) \frac{\partial \omega}{\partial x_{j}}\right]+\frac{\gamma}{v_{t}} \tau_{i j} \frac{\partial \bar{u}_{i}}{\partial x_{j}} \\
&-\beta \rho \omega^{2}+2\left(1-F_{1}\right) \frac{\rho \sigma_{\omega 2}}{\omega} \frac{\partial k}{\partial x_{j}} \frac{\partial \omega}{\partial x_{j}}
\end{aligned}
$$

The eddy viscosity is therefore defined as follows in eqs. (12).

$$
v_{T}=\frac{a_{1} k}{\max \left(a_{1} \omega S F_{1}\right)}
$$


460 where $a_{1}$ is a constant, $\omega_{T}$, is the turbulent dissipation, $S$ is the strain and $F_{2}$ is a

461 blending function which is set to 1 in the near wall and 0 in the free shear region.

The blending function $F_{1}$ is a function of the distance to the wall:

$$
F_{1}=\tanh \left(\arg _{1}^{4}\right)
$$

where

$$
\arg _{1}=\min \left[\max \left(2 \frac{\sqrt{k}}{0.09 \omega z} ; \frac{500 \mu}{z^{2} \omega}\right) ; \frac{4 \rho k}{\left(C D_{k \omega}\right) z^{2} \sigma_{\omega 2}}\right]
$$

with

$$
\left(C D_{k \omega}\right)=\max \left(2 \rho \frac{1}{\sigma_{\omega 2} \omega} \frac{\partial k}{\partial x_{j}} \frac{\partial \omega}{\partial x_{j}} ; 10^{-20}\right)
$$

464

Table 3

Model constants for inner 'wall' region

\begin{tabular}{lllll}
$\beta_{1}$ & $\beta^{*}$ & $\gamma_{1}$ & $\sigma_{k 1}$ & $\sigma_{\omega 1}$ \\
\hline .075 & 0.09 & 0.553 & 2.0 & 2.0
\end{tabular}

466

Table 4

Model constants for outer 'surface' region

\begin{tabular}{lllll}
$\beta_{2}$ & $\beta^{*}$ & $\gamma_{2}$ & $\sigma_{k 2}$ & $\sigma_{\omega 2}$ \\
\hline 0.0828 & 0.09 & 0.4404 & 1.0 & 1.17
\end{tabular}

468

\subsection{Boundary Conditions}

470

The following section outlines the boundary conditions used in the model.

\subsubsection{Pressure}

For the cylinder walls, a normal zero gradient $\left(\frac{\partial p}{\partial n}=0\right)$ was applied. For the

473 domain boundaries, a total pressure condition was imposed $\left(p+\frac{1}{2} \rho|\bar{u}|^{2}=0\right)$ allowing

474 for the pressure, $p$, to be computed from the velocity.

$475 \quad 3.2 .2$ Velocity

476 No-slip zero velocity condition was applied to the cylinder walls $(u=0)$, while for

477 the domain boundaries, a normal zero gradient ( $\frac{\partial u}{\partial n}=0$ ) was applied. 
At the cylinder walls, zero gradient was applied for the turbulent kinetic energy (

$\left.480 \frac{\partial k}{\partial n}=0\right)$ and for the specific turbulent dissipation, $\left(\omega=\frac{U_{f}}{\sqrt{\beta^{*}} \kappa y_{p}}\right)$ was applied at the

481 wall cells. The surface roughness of the cylinder walls was handled with eddy viscosity

$482 \operatorname{term}\left(v_{t}=\frac{\sqrt{\beta^{*}} \sqrt{k} y_{p}}{\log \left(E y^{+}\right)}\right)$which was derived from the wall function $\left(\frac{u}{u_{f}}=\frac{1}{\kappa} \log \left(E y^{+}\right)\right)$.

483 where $E=9.8$ for a smooth wall or $E=\frac{9.8}{\left[^{+} k_{s}^{\sin \left(0.4258 \cdot \log \left(k_{s}^{+}\right)-0.811\right)}\right.}$ when

$$
\left[\frac{k_{S}^{+}-2.25}{87.75}+C_{s} k_{S}^{+}\right]
$$

$2.25<K_{S}^{+}<90$ transitional roughness, or $E=9.8 /\left(1+C_{s} k_{S}^{+}\right)$for $k_{s}^{+}>90$ fully rough

wall. Parameter definitions are as follows; $k_{S}^{+}=k_{s} U_{f} / v$ is the non-dimensional roughness, $C_{\mu}$ turbulence model constant, $y_{p}$ distance to first calculation point from wall, $C_{S}$ roughness constant depending on the type of roughness, (set to 0.5 here) and $y^{+}$non-dimensional wall distance.

489

\subsection{Solution method}

The solution method is based on the open source library OpenFOAM (Open Field

492 Operation and Manipulation), (“OpenFOAM, The open source CFD toolbox,” 2018).

493 This is an open source, object-orientated C++ library for numerical simulations of fluid 494 dynamics.

495 The numerical model applied was a modified version of the standard transient,

496 incompressible PIMPLE algorithm that is a combination of the PISO-algorithm, (Issa,

497 1986) and the SIMPLE-algorithm (Caretto et al., 1973) as part of the solver

498 pimpleFoam in OpenFOAM, which includes an oscillating body force term

499 representative of the wave flow field. This body force term was used to model periodic

500 oscillations. The numerical integration in time was based on the PIMPLE-algorithm

501 using an Euler backward time integration. The Gauss linear Upwind (LUD) resolved the 502 convective terms, while central differences was used for diffusive terms. This leads to a 503 spatial resolution close to second order. The pressure equation that can be derived from 
504

505

506

507

508

509

510

511

512

513

514

515

516

517

518

519

520

the continuity equation was solved using Generalised geometric/algebraic multi-grid solver (GAMG), while the momentum equations and the turbulence equations were solved using preconditioned bi-conjugate gradient solver (PBICG). As preconditioner the incomplete LU (DILU) was used for the momentum and turbulence equations.

\subsection{Meshing strategy}

Model geometries for the jacket and monopile foundations with secondary boat landing structures were created as presented in the previous sections. Rotated geometries to model the effect of varying wave incidences (typically in $30^{\circ}$ intervals) were also created, along with jacket and monopile structures without secondary structures.

Model meshes were created using the OpenFOAM automated meshing library snappyHexMesh (sHM). This produced 2D meshes with a majority of hexahredal elements. Boundary layer elements were added in order to apply rough wall boundary conditions. The panel a) in Figure 8 presents the overall mesh for the monopile foundation with secondary structures rotated at $60^{\circ}$, while b) and c) are close-ups of the cylindrical structures.

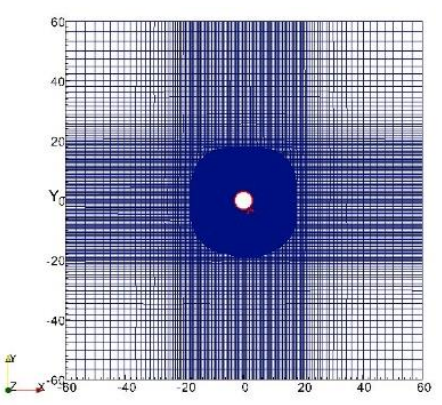

a

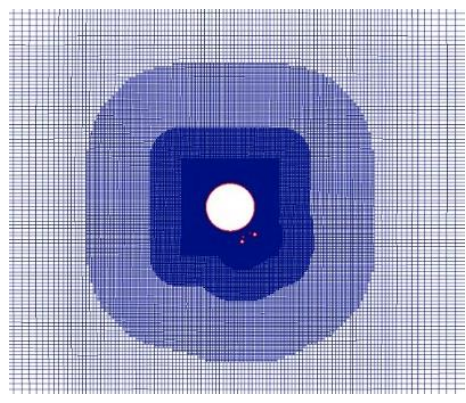

b

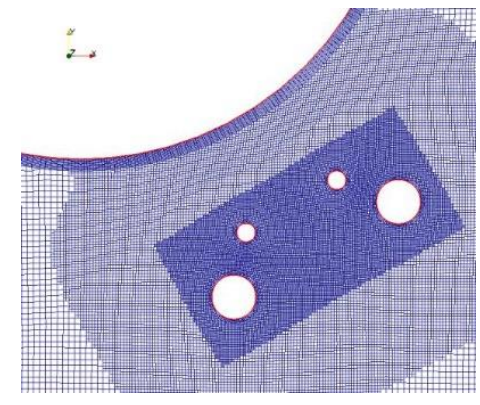

$\mathrm{c}$

521 Figure 8 Mesh for $6 m$ diameter monopile with secondary structures at $60^{\circ}$ rotation,

$522 k s=0.0045 \mathrm{~m}$. a) View of full domain. b) Close-up of the monopile and secondary

523 structures with graded refinement. c) Close-up of secondary structures with partial view 524 of monopile with boundary layer elements visible.

\section{Test with a monopile}

In order to test and validate the experimental and numerical methods several test cases were carried out for a circular cylinder alone, i.e. without secondary structures. 
529 This included qualitative assessment of the inline force time series and comprehensive

530 comparison of obtained force coefficients to well-known results in the literature.

531 Figure 10, Figure 11 and Figure 12 shows force time series from the three

532 numerical calculations that can be compared to results presented in (Sarpkaya, 1976).

533 The shape of the force times series can also be seen to have qualitatively resemblance

534 with results presented in (Saghafian et al., 2003). The figures show examples of inline

535 force in the range for KC-number 10 to 30. It can be observed from the figures that

536 there was a qualitative resemblance of the shape of the force profiles with changing $K C$,

537 between the experiments and the CFD models. As the $\mathrm{KC}$ number increased the number

538 of vortex shedding are expected to increase as well, which leads to additional

539 fluctuations in the force signal. An example of the vortex shedding is given in Figure 9.

540 In this case the $\mathrm{KC}$ was 20 and therefore several vortex shedding per oscillation were

541 generated.
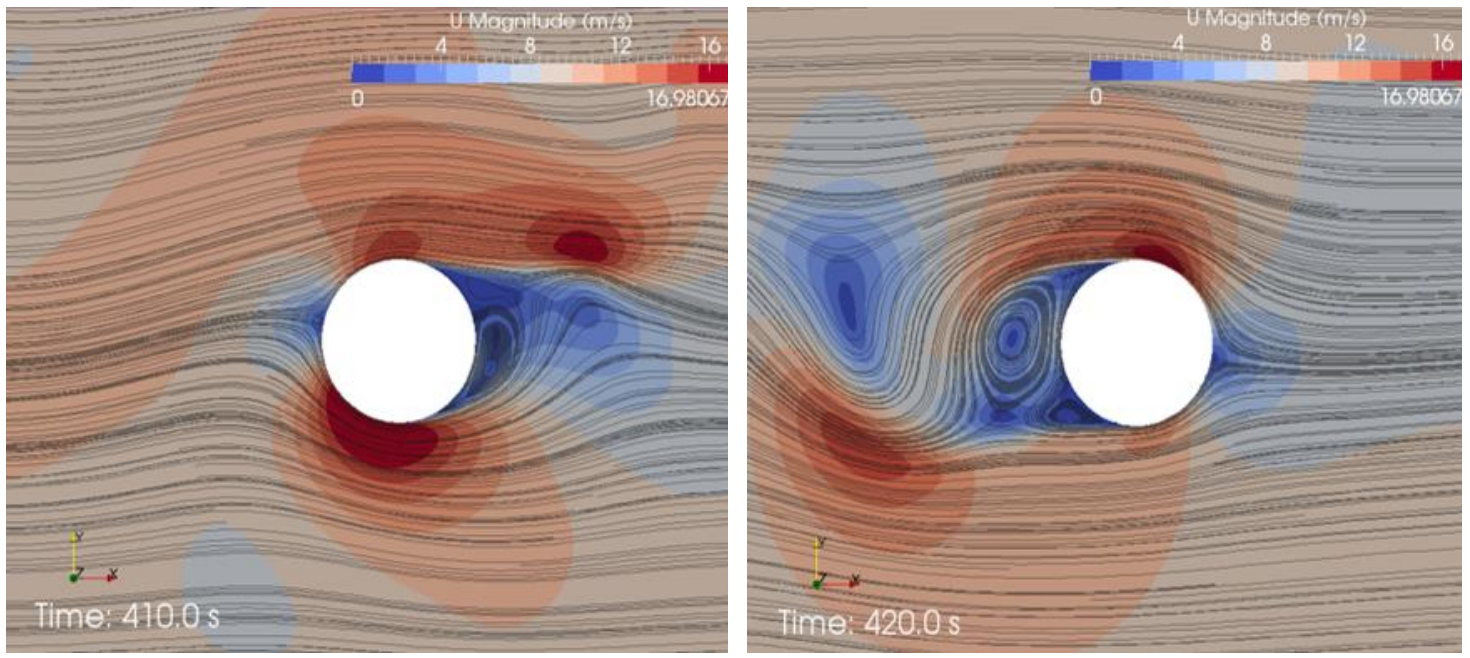

542

543

544

545

546

547

548

549

550

Figure 9 Illustration of the vortex formation in a case with $K C=20$.

These fluctuations were compared to results presented in (Sarpkaya, 1976) and we found that they qualitatively had the same shape. We also clearly see that the Morisonforce model did not resolve the fluctuations due to the vortex sheddings. Both measured, and modelled forces have typical higher maximum load forces than predicted with the Morison model. This is partly related to the least squared fit that is the common choice for estimation of force coefficients, and partly due to inadequate representation of the vortex shedding mechanisms in a Morison force model. 


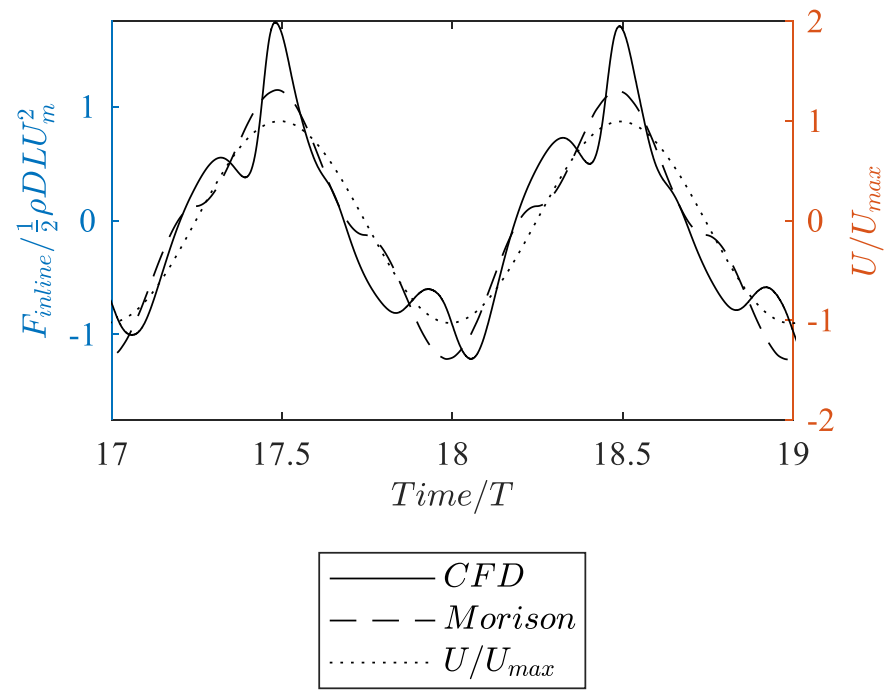

551

552

553

554

Figure $10 C F D$-model, $K C=10.5 C F D$-model, $k_{s} / D=0.01$. Resembles result presented in (Sarpkaya, 1976), in his fig. 24i. In his case $K C=12$.

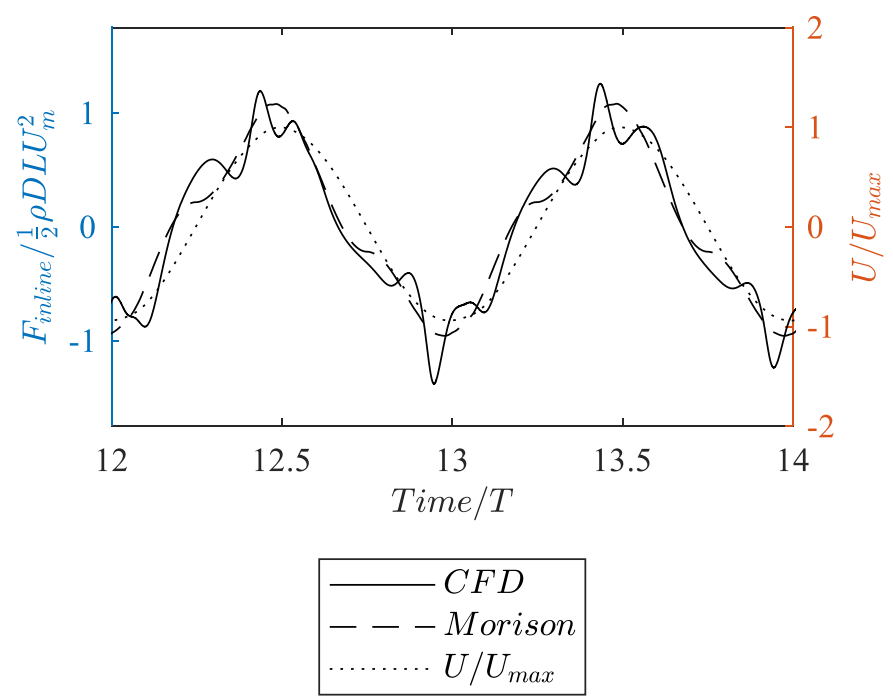

556 Figure 11 CFD-model, $K C=15.7, k_{s} / D=0.01$. Resembles result presented in 


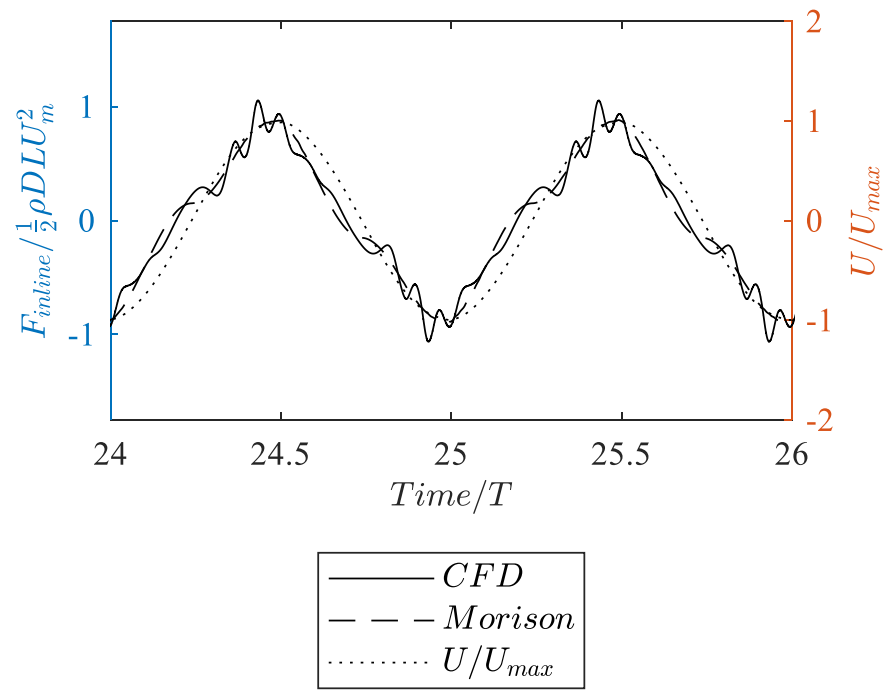

559

560

561

562

563

564

565

566

567

568

569

570

571

572

573

574

575

576

577

578

579

580

Figure $12 K C=26.2, k_{s} / D=0.001$. Resembles result presented in (Sarpkaya, 1976), in his fig. 24e. In his case $K C=25.4$.

Figure 13 shows two examples of time-series of measured and modelled inline forces. The general shape of the force time series appears the same. In the measured force time series more fluctuations were observed for the low $\mathrm{KC}$ number, $K C_{\text {mea }}=7.7$ compared to the CFD results. For the large KC-number, $K C_{C F D}=31.3$, the fluctuations from the CFD calculations appear stronger compared to the measured time-series. This might stem from the use of URANS in 2D, which will be further discussed later.

Experiments for loads on rough cylinders in oscillatory flows were conducted and presented by (Sarpkaya, 1976) The results of that study cover a wide range of KCnumbers, where the interval $\mathrm{KC}=[20,30,40]$ and a relative surface roughness of $k / D \square 1.25 \cdot 10^{-3}$ is relevant for the present study and the comparison. Further, additional data from (Justesen, 1989) was included in the comparison, even though the relative surface roughness was larger, $k / D \square 3.0 \cdot 10^{-3}$. Figure 14 shows the comparison of $C_{D}$ and $C_{M}$ over the tested range of the KC-number for a monopile alone. Firstly, it can be observed that the overall results of the $C_{D}$ and $C_{M}$ of the physical model and those of the CFD model were in good agreement, and therefore the validation of the CFD model seems confirmed in the case of the cylinders without secondary structures. Clearly, for both $C_{D}$ and $C_{M}$ scatter exists, which may be caused by the experimental uncertainties. 

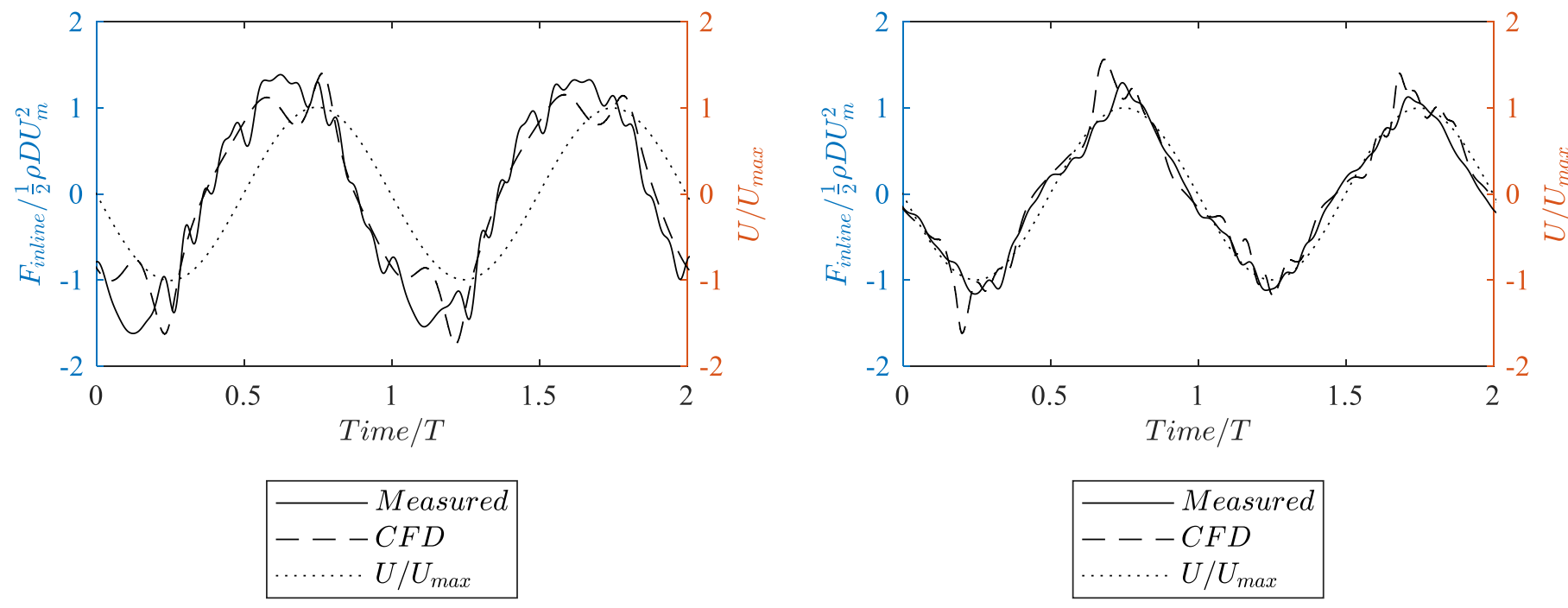

581 Figure 13 Two examples of comparisons of force time-series from physical (measured) 582 and $C F D$ models. The left panel had $K C_{\text {mea }}=7.7$, and $K C_{C F D}=7.9$. The right panel had $583 K C_{\text {mea }}=31.8$, and $K C_{C F D}=31.3$. The relative roughness $w a s$ in the order,

$584 \quad k_{s} / D \square 1.25 \cdot 10^{-3}$.

585

586 Force coefficients for both the experimental and numerical analyses showed the 587 same behaviour as reported in (Sarpkaya, 1976), and (Justesen, 1991). The drag

588 coefficient, $C_{D}$, increased from around 0.5 to around its maximum value around 1.75 at

$589 K C=10-11$. Then the $C_{D}$ coefficient decreases and becomes almost constant as $K C$

590 increases from 20 to 40 . The inertia coefficient decreased from its asymptotic value 2 at

$591 K C \approx 0$ to it minimum value around 0.75 based on the CFD model and 0.5 literature

592 values from (Justesen, 1991), for a $\mathrm{KC}$ in the range 10-15. From a $K C$ equal to and

593 larger than 20 the $C_{M}$ was constantly in the range 1.3-1.5. 

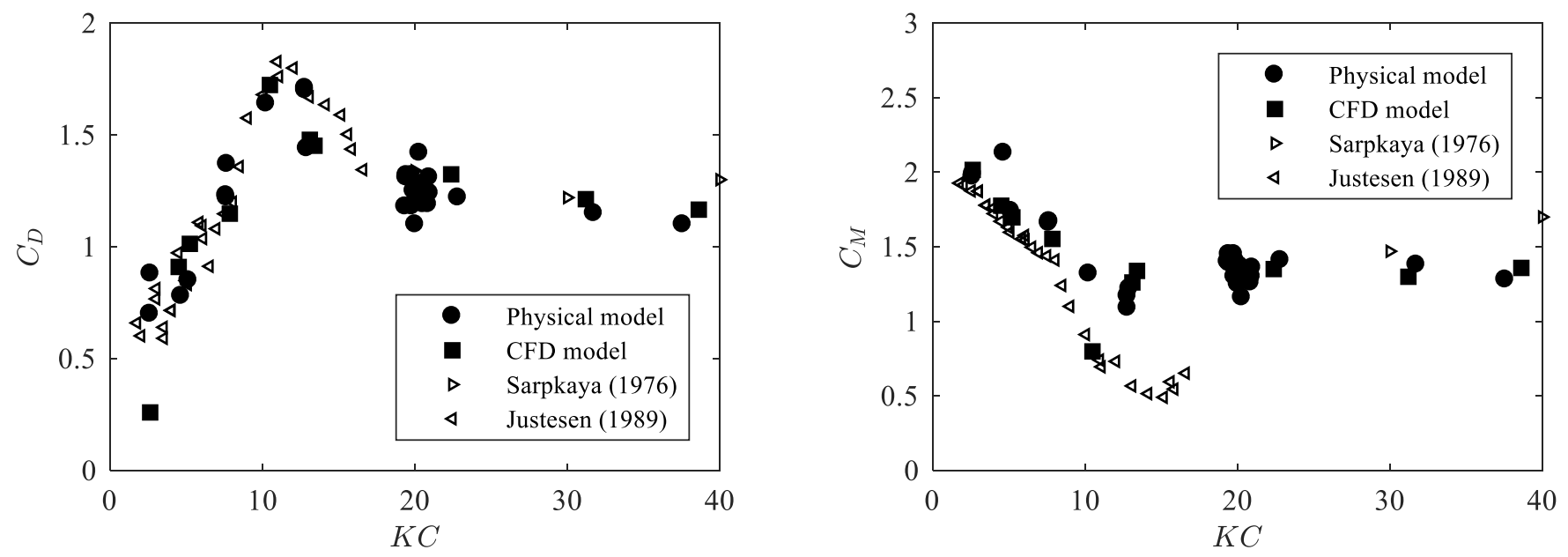

595 Figure 14 The variation of $C_{D}$ and $C_{M}$ over the tested range of the $K C$-number for the

596 monopile and jacket leg model without secondary structures attached. The relative

597 roughness was in the order, $k_{s} / D \square 1.25 \cdot 10^{-3}$ except for the case of (Justesen, 1989),

598 where it was $k_{s} / D \square 3 \cdot 10^{-3}$.

599

600

The force coefficients were found to be in good agreement with the earlier studies

601 and also between the physical tests and numerical calculations. CFD analyses based on

602 a URANS model is known to have difficulties in predicting the drag crisis in pure current, see for instance (Wen and Qiu, 2017). In (Saghafian et al., 2003) similar conclusions were found for pure current, while numerical simulations for an oscillatory outer flow showed better agreement with measurements. In their case the surface of the circular cylinder was smooth while it was rough in all our cases. The main problem for the URANS models have often been linked to inadequate capabilities in predicting the transition from laminar to turbulent boundary layer, which results in difficulties in finding the correct position of the separation points. The position of separation points has a significant influence on the drag force and therefore have to be modelled correct

611 to find an accurate answer for the drag force.

612 URANS has difficulties in predicting the drag crisis in pure current, but we find 613 that there were two reasons for the good agreement between measurements and CFD 614 based on URANS modelling in our study. First of all, the outer flow was periodically 615 oscillating and not a steady current. The dependence of the Reynolds becomes less 616 abrupt as in pure current as reported in the literature (Justesen, 1989; Sarpkaya, 1986, 617 1976). Secondly, all cases were for Reynolds high numbers in the CFD calculations and 618 trip wires were used in the physical experiments. The trip wires ensured a turbulent 
619 boundary layer, and as earlier discussed, which was seen to results in less dependence

620 on the Reynolds number. Often trip wires are believed to produce flow conditions valid

621 for full scale. For high Reynolds flows we see less dependence of the force coefficients

622 on the Reynolds number, while the relative roughness $k / D$ has some influence. In

623 (Bjørke et al., 2018) the forces on square cylinders were analyses based on a similar

624 CFD model, and they also found a good agreement between physical model tests and

625 CFD analyses in oscillatory flow. In their cases edges of square cylinders fixated the

626 position of the separation point making it independent on the Reynolds number within

627 the range.

628

629

630

631

632

633

634

635

636

637

638

639

640

641

642

643

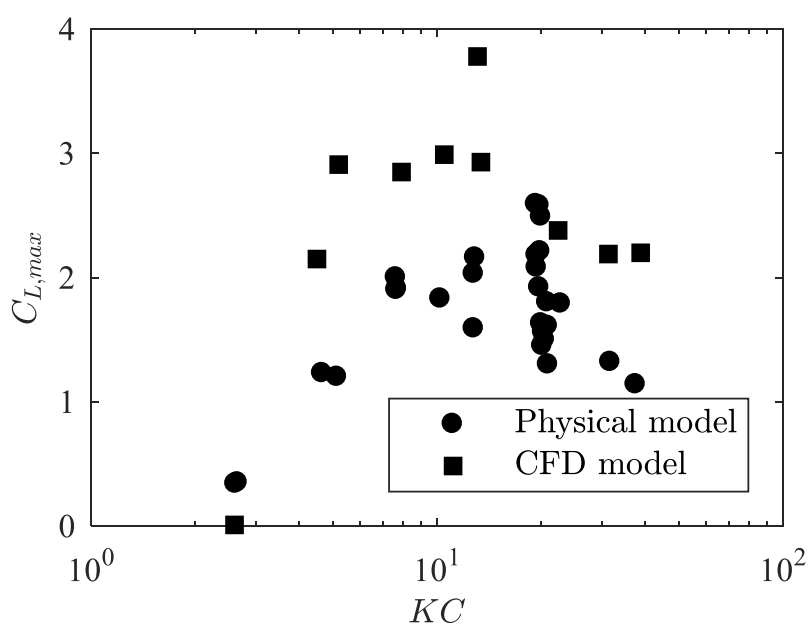

Figure 15 The Maximum lift force coefficient, $C_{L, \max }$, for over the tested range of the $K C$-number for the monopile and jacket leg model without secondary structures attached. The relative roughness was in the order, $k_{s} / D \square 1.25 \cdot 10^{-3}$.

Figure 15 shows the variation of the $C_{L, \max }$ coefficient over the tested range of the $\mathrm{KC}$-number for both the monopile and the jacket leg models in the case of a single plain cylinder. Clearly, as expected, the lift force approached zero as $K C$ go to 0 , here unseparated flow exists and hence no lift force occurs. This trend was well preserved in both the data from the physical and the CFD model. As the $\mathrm{KC}$-number increases the $C_{L, \max }$ increased, with a maximum around $\mathrm{KC}=10-20$. As the $\mathrm{KC}$-number increased further the $C_{L, \max }$ decreased. The CFD model overestimated the lift coefficient in relation to the physical model; however, the estimated values are still in the same order of magnitude as the data of a smooth cylinder.

The enhanced lift force experienced in the CFD model may also be attributed to the two-dimensional set-up. The vortices will typically be larger in two-dimensional 
644 models (like the present) and have difficulties in breaking down to smaller vortices.

645 This might induce a lower pressure over larger areas leading to an enhanced lift. It

646 should also be noted that the exact maximum obtained from the measured force time

647 series in the physical model was slightly biased with the filtering made in the post-

648 processing of the measured force signal. Here, the local maxima may be reduced

649 slightly, however, given the reasonable agreement with the previous works of

650 (Sarpkaya, 1976) this effect seems negligible.

651

6525 Results

653 Results from physical and numerical experiments will be presented in this chapter.

654 It includes examples on the force time-series, the dependence of the force coefficients

655 on $\mathrm{KC}$ number and orientation of the secondary structure to the main flow direction.

656

\section{$657 \quad 5.1$ Monopile with secondary structures}

658 Figure 16 show the force time-series from the physical and numerical tests where

$659 \mathrm{KC}=5.2$. In the results from the physical model experiments, the main periodic signal

660 was overridden small short oscillations. This might stem from noise originating from

661 the use of a carriage, which the standard filtering procedure was unable to resolve. Still,

662 the comparison between the measured and modelled force time series showed a good

663 agreement for the purpose estimation of force coefficients for the Morisons equation.

664 For small KC-numbers the forces were smaller in the experiments and therefore the

665 results become more sensitive to the filtering procedure. The CFD force time-series had

666 approximately the same behaviour as in the experiment. Both cases were dominated by

667 the inertia part as the maximum force was found close to the maximum acceleration.

668 


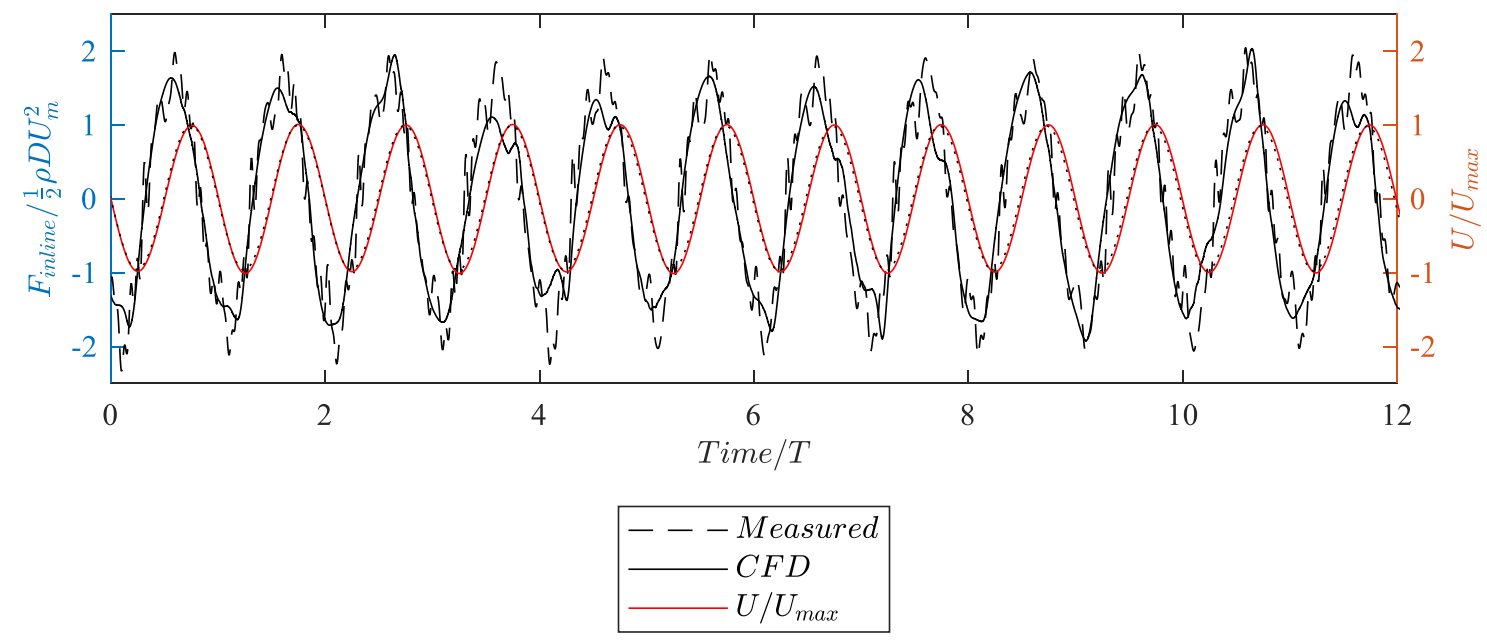

669

670

671

672

673

674

675

676

677

678

679

680

681

682

683

684

Figure 16 Time series of inline forces for $K C=5.2, k_{s} / D=1.25 * 10^{-3}$. Mono-pile with secondary structures at $0^{\circ}$ orientation.

In Figure $17 K C \approx 13$ that is in the intermediate range where both the drag an inertia terms in the Morisons force model contribute substantial to the force. The shape of the force time-series was overall the same in the physical measurements and the CFD calculations. The force had or was close to have a local maximum at maximum acceleration, but the maximum force was found close to maximum speed. However, there seems to be a tendency to larger subharmonic contributions in the CFD results.

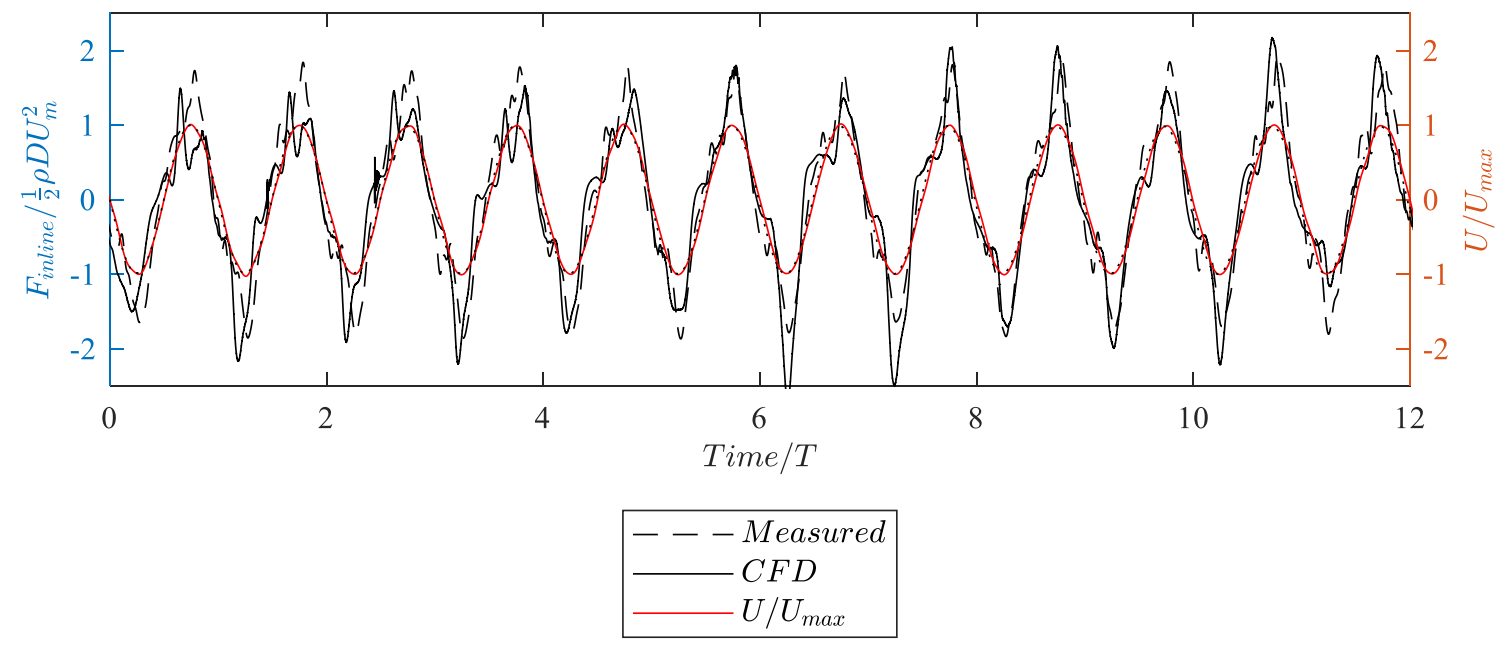

Figure 17 Time series of inline forces for KC-numbers: $K C_{C F D}=13.1, K C_{\text {Phys. }}=12.9$, $k_{S} / D=1.25 \cdot 10^{-3}$. Mono-pile with secondary structures at $0^{\circ}$ orientation.

Figure 18 shows the force time-series for jacket-leg with secondary structures at $0^{\circ}$ orientation. With a relative high, $\mathrm{KC}$-number, $\mathrm{KC} \approx 31-32$, the forces will be drag 
685

686

687

688

689

690

691

692

693

694

695

696

697

698

699

700

701

702

703

704

705

706

dominated. The maximum force was in both physical experiments and in the CFD calculations located close to the maximum speed. In the CFD results fluctuations in the force were present especially when the secondary structure was on the lee side of the main circular cylinder. The largest cylinders in the secondary structure could interfere with the vortex shedding from the main structure, as they were located outside the shadow zone of the main cylinder.

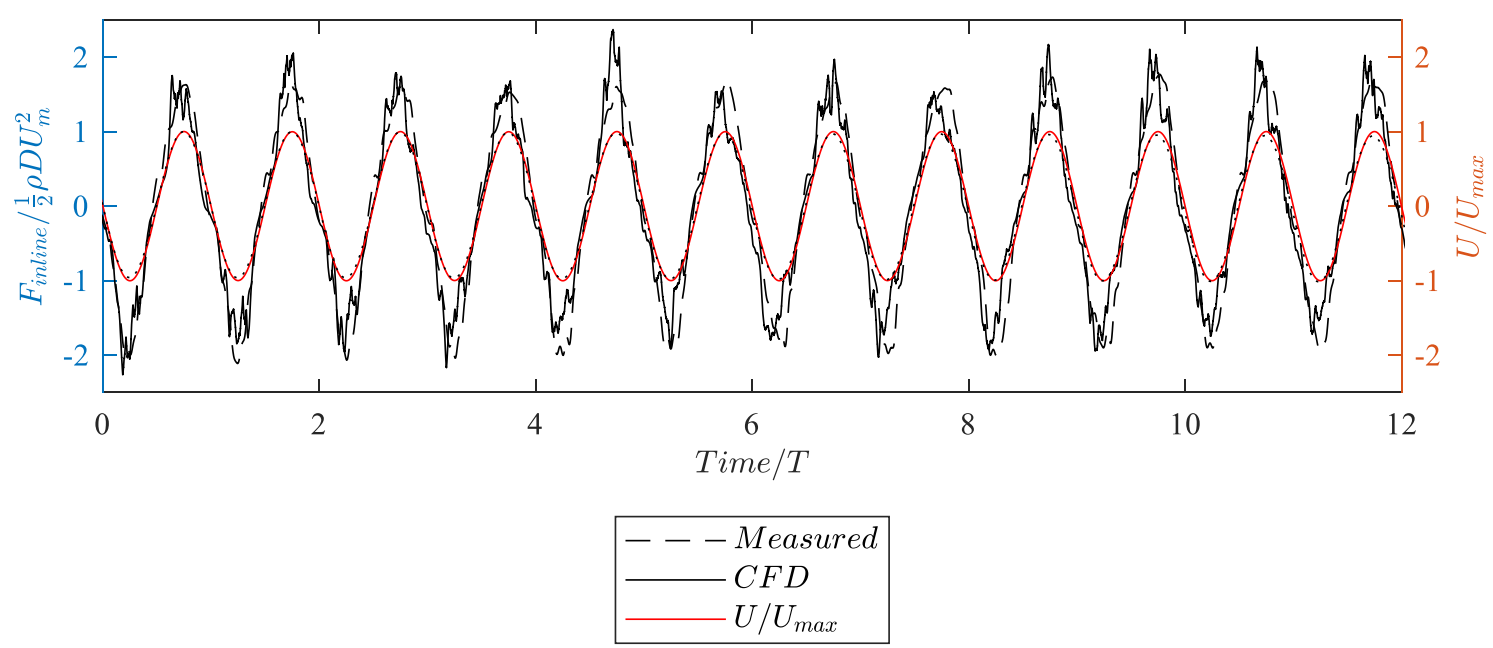

Figure 18 Time series of inline forces for $K C$-numbers: $K C_{C F D}=31.3, K C_{\text {Phys }}=32.3$, $k_{s} / D=1.25 \cdot 10^{-3}$. Jacket-leg with secondary structures at $0^{\circ}$ orientation.

Time series with 0 degrees and 90 degrees versus for jacket leg with secondary structures are shown in Figure 19. The maximum forces appeared slightly larger for 90 degrees orientation of the secondary structures. The force time-series from especially the CFD calculations was at 0 degrees orientation slightly asymmetric compared to the 90 degrees orientation. The force-time series from the CFD-calculations appeared more fluctuating than the force time series from the physical experiments. The reason for the difference in strength of the fluctuations was not clear, but could be explained by the filtering of the signal in the experiments at $10 \mathrm{~Hz}$, whereby some of the fluctuations might have been filtered out. Another explanation could be related to shortcomings of the numerical model, where the 2D URANS simulations have shown to give larger fluctuations. 


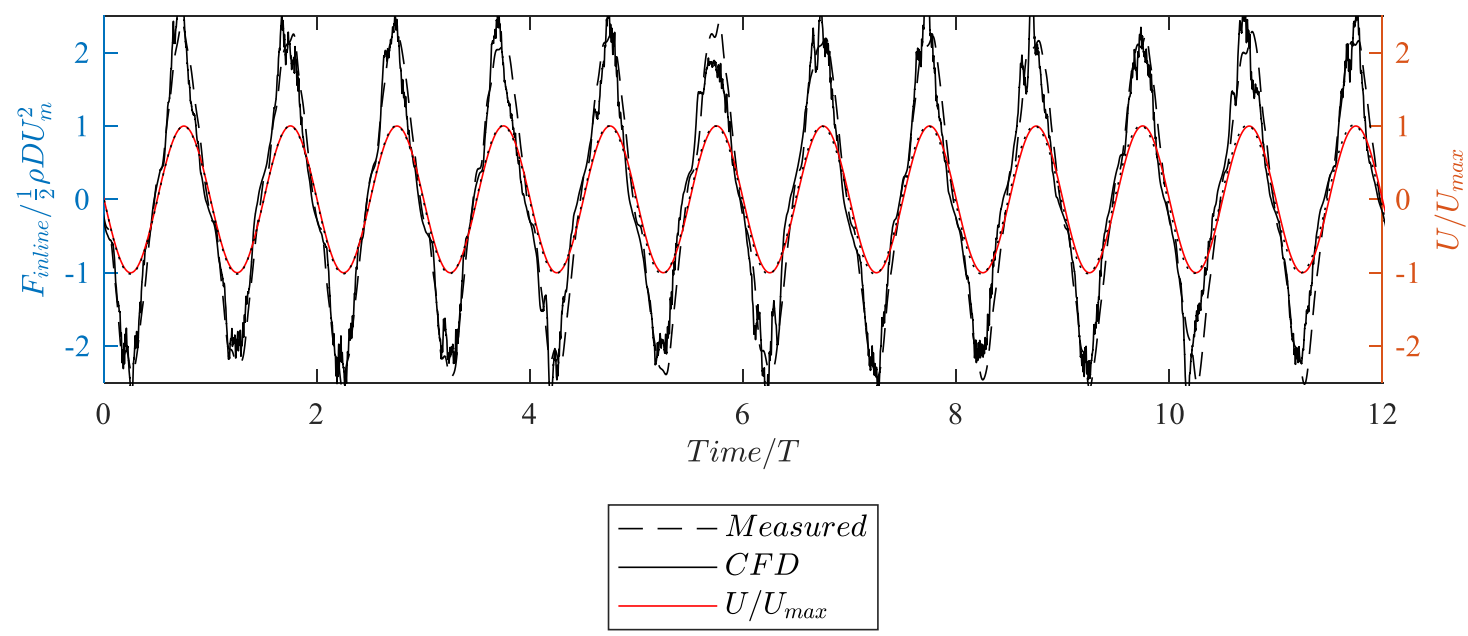

708

709

710

711

712

713

714

715

716

717

718

719

720

721

Figure 19 Time series of inline forces for $K C$-numbers: $K C_{C F D}=31.3, K C_{\text {Phys }}=32.3$, $k_{\triangleleft} / D=1.25 \cdot 10^{-3}$. Jacket-leg with secondary structures $90^{\circ}$ orientation.

\subsection{Effect of orientation of the secondary structure on force coefficients} Figure 20, Figure 21, Figure 22, and Figure 23 show the variation of the $C_{D}$ and $C_{M}$ with the orientation of the secondary structure, $\theta$. The individual figures represent different $K C$-number regimes. Note that for each plot the $K C$-number regime was expected to be sufficiently narrow to assume that the flow regime for each plot was the same in the physical model tests as in the CFD model tests. For the drag force coefficient, $C_{D}$, it was clear that for each of the tested $K C$-numbers the drag increased almost linearly with increasing $\theta$ for both the physical and CFD model results. This behaviour was expected as the "exposed" part of the overall structure that immediately was in contact with the main body of the flow increased with increasing $\theta$, and in turn this increased the drag force on the structures.

At $\theta=0^{\circ}$ the value of $C_{D}$ was noticeably at the same order of magnitude or lower as in the case of a cylinder with no secondary structures. This is attributed to the secondary cylinders affecting the flow around the main cylinder similarly to a splitter plate, where the effect of the vortex shedding on the lee-side of the cylinder is damped, and in turn the drag decreases.

As $\theta \rightarrow 30^{\circ}$ the drag increased so $C_{D}$ was of the same magnitude as in the case where no secondary structures are attached, and the $C_{D}$ further increases as $\theta=90^{\circ}$. Further to this, when examining the increase in $C_{D}$, one should bear in mind that some parts of the increase were inherent in the least-square-fit method applied in the present 
731 investigation, where the diameter, $D$, and the cross-sectional area, $A$, refer to the main 732 pile geometry.

733 For the drag force coefficient, $C_{D}$, it was clear that for each of the tested $K C$ -

734 numbers the drag increases with increasing $\theta$ until it reached a maximum around $\theta=60$

735 after which it stabilized or decreased slightly. This effect was mainly caused by the

736 "exposure" of the individual structural elements of the secondary structures to the main

737 body of the flow being largest at this plan view orientation. Clearly, the projected length

738 of the individual structural elements onto the transverse plane (y-plane) was largest at $\theta$

$739=60^{\circ}$.

$740 \quad$ For the inertia force coefficient, $C_{M}$, the physical and the CFD model results

741 agreed well for the lower $K C$-numbers. With increasing $K C$-number slight discrepancies

742 were observed.. According to (Sumer and Fredsøe, 2006) the transverse vortex street

743 appears in the range $7<K C<13$, where the wake consists of a series of vortices

744 convecting out to one side of the cylinder. This effect/discrepancy can also be seen for

745 the same interval of the $K C$-number in the results of the cylinders without secondary

746 structures illustrated in the right panel in Figure 14. Apparently, $C_{M}$ was more effected

747 by this than $C_{D}$ was.
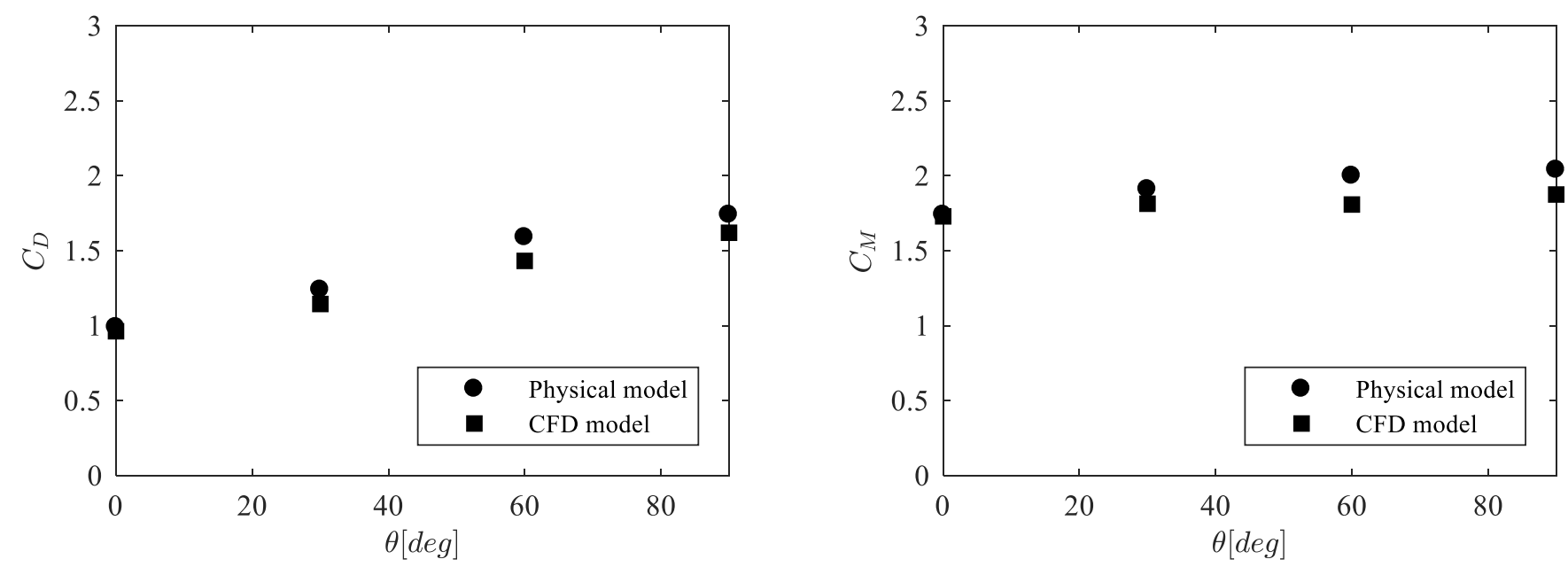

Figure $20 \mathrm{KC}=5.2$. Monopile with secondary structures

749

750 

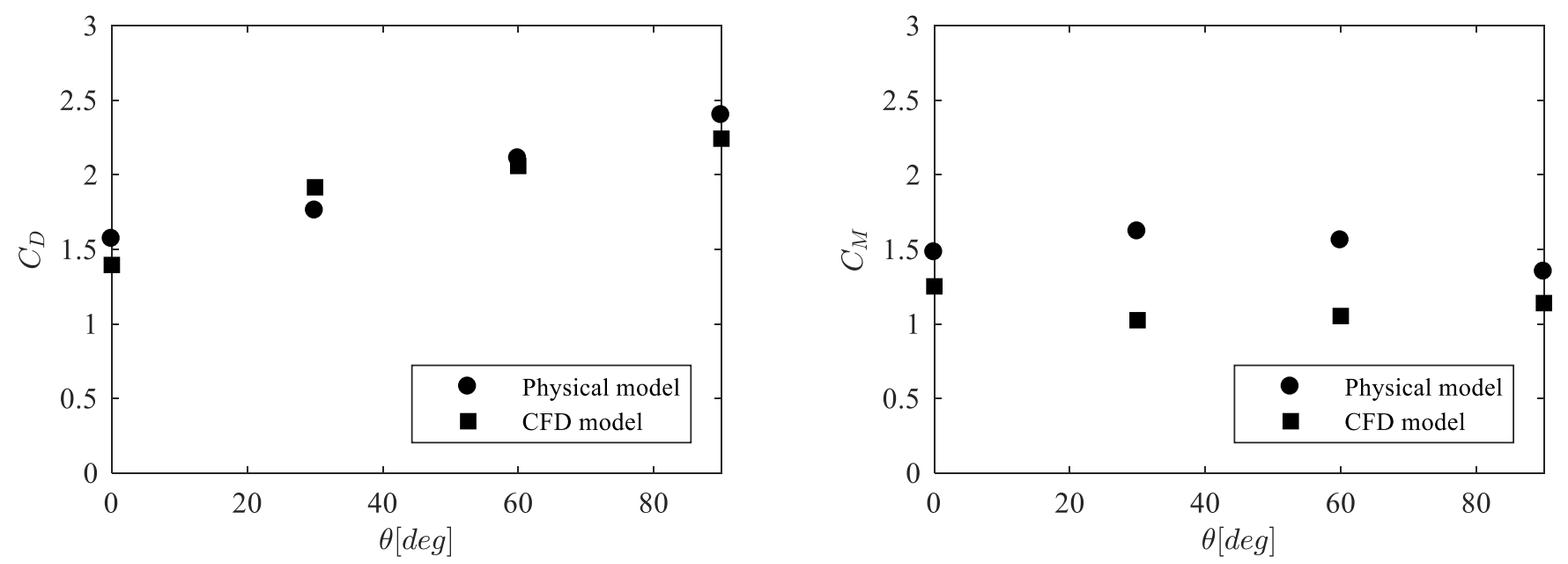

751 Figure $21 \mathrm{KC}=10$, Monopile with secondary structures

752
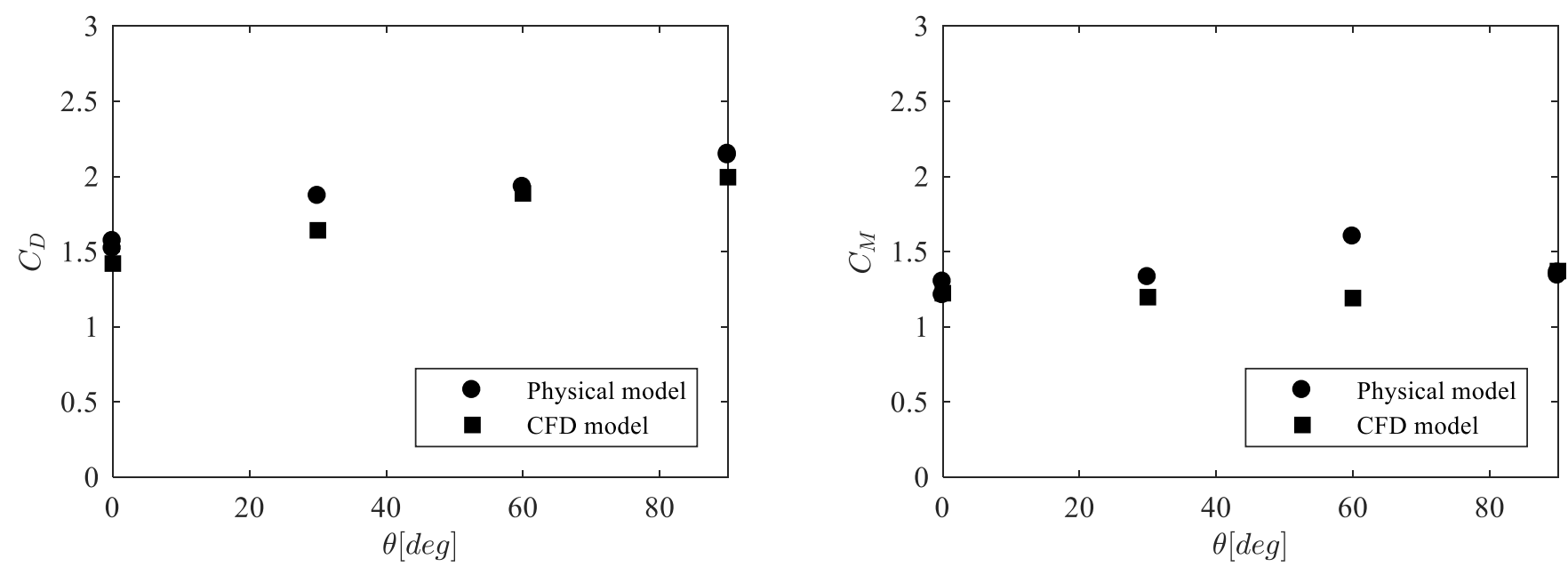

753 Figure $22 \mathrm{KC}=13$, Monopile with secondary structures

754

755 

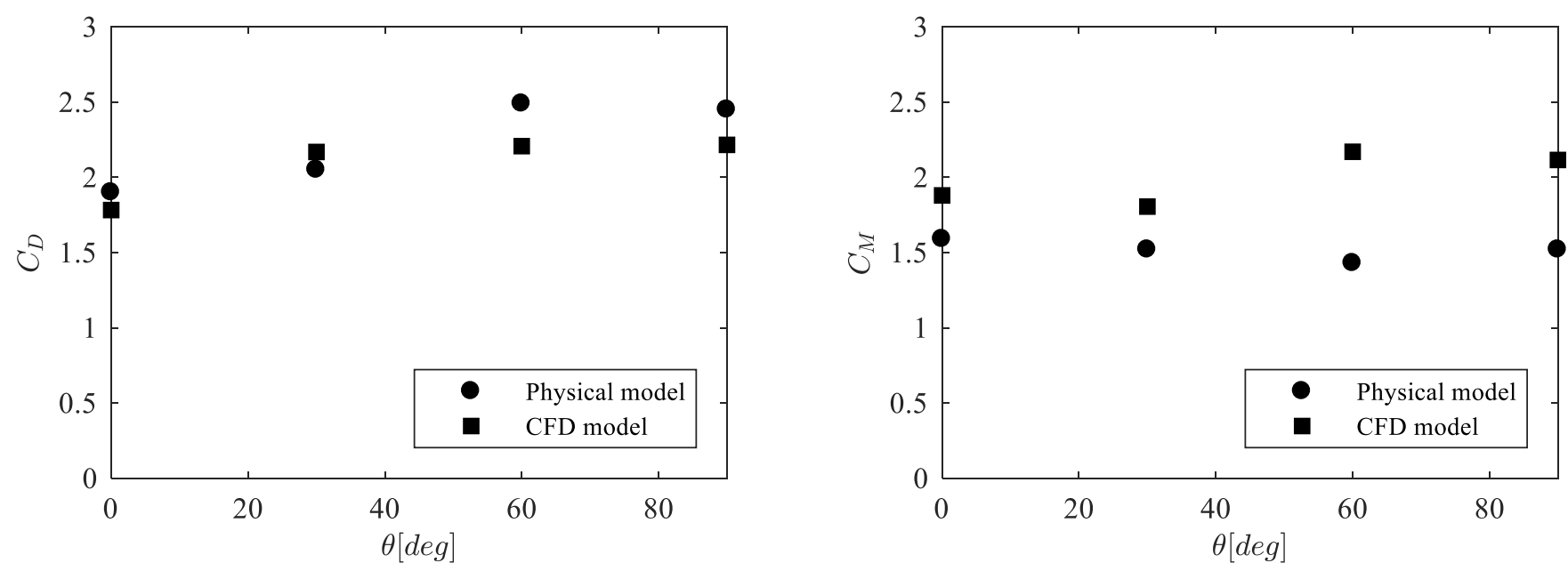

Figure $23 \mathrm{KC}=31$, Jacket leg with secondary structure

The effect of the plates shown in Figure 5 was $10 \%$ increase of the $C_{D}$ coefficient when the orientation was $\theta=90^{\circ}$. As the $K C$-number increased the discrepancy between

760 the $C_{D}$ values obtained in the physical and the CFD model decreased. A good agreement was achieved for $K C$ in the order of 31 as shown in Figure 23.

\subsection{Variation of force coefficients with $\mathrm{KC}$}

Figure 24 displays the variation of the $C_{D}$ and $C_{M}$ with $K C$ for different $\theta$ values $\left(0^{\circ}, 30^{\circ}, 60^{\circ}\right.$ and $\left.90^{\circ}\right)$. The figure generally support the discussions on the results of $C_{D}$ and $C_{M}$ made in the previous section, which are further detailed in the following. The good agreement between the $C_{D}$ values obtained from the physical and the CFD models, explained in the previous section is also present in Figure 24. Here the slight discrepancies in $C_{D}$ are mainly observed at very low $K C$-numbers $(\mathrm{O}(2))$, which are preserved from the results of the cylinders without secondary structures presented in section 4. Similarly, the discrepancy in $C_{D}$ for this range of the $K C$-number, where the inertia part of the force dominates, will not affect the total load (consisting of drag and

773 inertia contribution) response significantly. Hence the discrepancy seems negligible.

774 The $C_{D}$ values obtained in the physical model are generally slightly higher than those in 775 the CFD model. This can be attributed to the form drag and the skin friction exerted by 776 the oscillating flow on to the secondary cylinders-carrying plates in the physical model.

777 The lowest markers in Figure 24 represent CFD results for each orientation. 

model results gave confidence in the simulations. The previously mentioned

780 discrepancy in the $C_{M}$ coefficient around $K C \sim 10$ is visible in the present Figure 24

781 One can observe that this discrepancy is largest for the orientations $\theta=30^{\circ}-60^{\circ}$ in

782 Figure 24. No clear explanation for this has been offered in the present investigation.
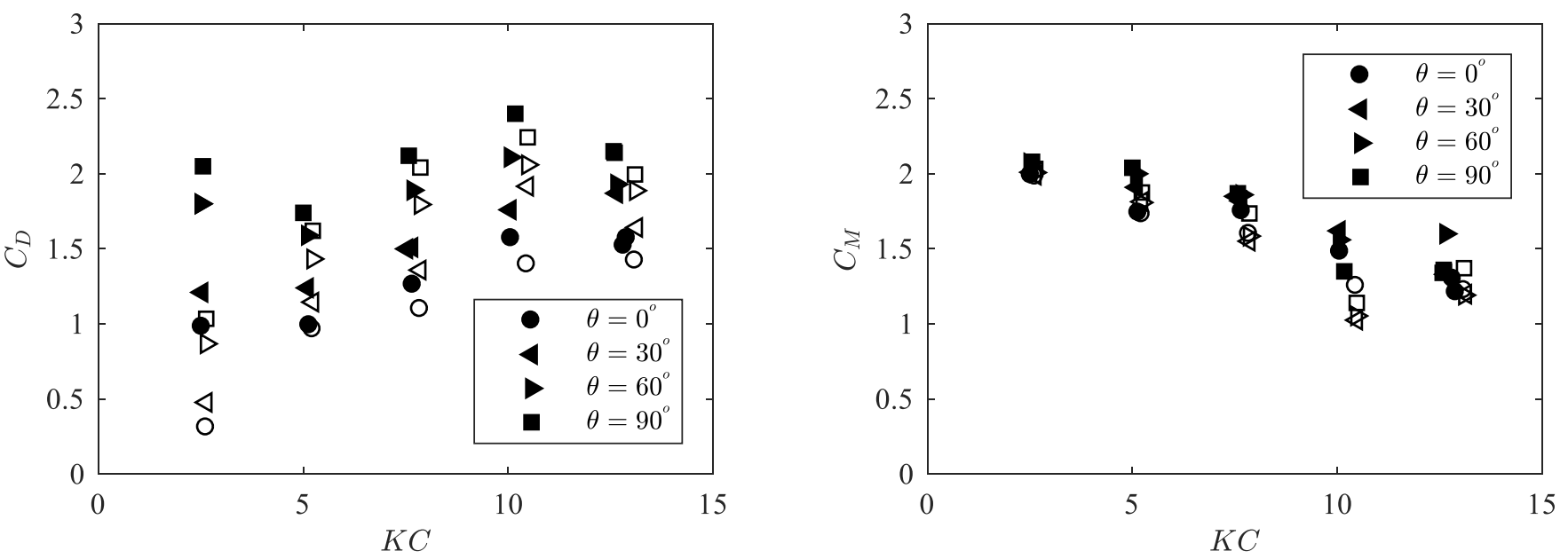

783 Figure 24 Variation of force coefficients with KC-number for 4 different orientations of

784 the secondary structure. Structure: Monopile with secondary structures. Filled symbols: Physical model tests. Empty symbols: CFD test.

786

Figure 25 displays the variation of the $C_{D}$ and $C_{M}$ versus 4 orientations of the secondary structure attached to the jacket leg. For the drag force coefficient, $C_{D}$, it was clear that for each of the tested KC-numbers the drag increased with increasing $\theta$ until it reached a maximum around $\theta=60^{\circ}$ after which it stabilized or even decreased slightly. This effect could be caused by the "exposure" of the individual structural elements of the secondary structures to the main body of the flow being largest at this plan view orientation. Clearly, the projected length of the individual structural elements onto the transverse plane (y-plane) was largest at $\theta=60^{\circ}$. The general agreement between the physical and the CFD model result of $C_{D}$ was reasonable. For the lower range of the KC-numbers $(\mathrm{KC} \sim 4-5)$ the $C_{D}$ value was larger in the physical model, which could be explained in conjunction with the effect of supporting plates shown Figure 5. Here, as previously explained in the case of the monopile model, the carrying plates exert a contribution to the overall drag, which was not simulated in the CFD model. The effect

800 of the plates on the $C_{D}$ relative to a single plain cylinder was up to $10 \%$ at $90^{\circ}$. It should 801 be noted that for small KC-numbers the drag part of the total force in the Morison 
802

803

804

805

806

807

808

809

810

811

812

813

814

815

816

817

818

819

820

821

822

823

824

825

826

827

828

829

830

831

832

833

834

equation is small, typically the drag contribution to the maximum load is negligible when the $\mathrm{KC}$ number is smaller than 5-6. Therefore, the estimation of the drag force coefficient will be attached with some uncertainty, without affecting the force estimated from the Morison formula. As the $K C$ number increased the discrepancy between the $C_{D}$ values obtained in the physical and the CFD model decreased, and the agreement was good at $K C=30-40$, where the drag contribution to the maximum force dominates.

The inertia coefficient, $C_{M}$, remained practically constant when changing the orientation $\theta$. This was in agreement with the results of $C_{M}$ obtained in the monopile case explained in the previous subsections. For the inertia force coefficient, $C_{M}$, the physical model and the CFD model results agreed well in the range of the KC-number 13 - 23. For larger KC-numbers discrepancies existed in the prediction of $C_{M}$ as can be observed in Figure 25, which is in line with the observation discussed above, as the drag part will dominate the force for KC numbers larger than 30-40. Also observe the general offset in between $C_{M}$ values measured in the physical model relative to the ones produced in the CFD model at $K C=4.4-4.7$. Here it seems that the physical model overestimated the $C_{M}$ value, and this was supported by the higher $C_{M}$ value for the same flow climate in the case of the cylinder with no secondary structures, as shown in Figure 14. Here the $C_{M}=2.1-2.13$ which was slightly higher than expected for this $\mathrm{KC}$ range considering the specific relative surface roughness when observing Fig. 4.19 in (Sumer and Fredsøe, 2006). Discrepancies can be also attributed to the CFD calculations modelling the turbulence and vortex structures as strictly two-dimensional, which will not be the case in nature/full scale nor in the physical model. The effect is not fully explained by this study. However, asymptotic theory based on potential theory needs a correction for the boundary layer to include viscous effects that could increase the $C_{M}$ number due to boundary layer velocity deficit at small $\mathrm{KC}$ values, as reported in (Bearman et al., 1986). It is not fully clear if a similar effect is introduced when using 2D CFD where the vortex shedding cannot break up in 3D structures. This could for instance lead to a change in boundary layer thickness and therefore a difference of $C_{M}$ between CFD and physical model experiments. The presented discrepancies between the physical model and the CFD model results for $C_{D}$ and $C_{M}$ were favoured by the relative influence of the drag and inertia force over the KC regime. (Sumer and Fredsøe, 2006) reported the range of the $\mathrm{KC}$-number $0<\mathrm{KC}<20-30$ as the inertia dominated regime, while $\mathrm{KC}>$ $20-30$ as the drag dominated regime. 

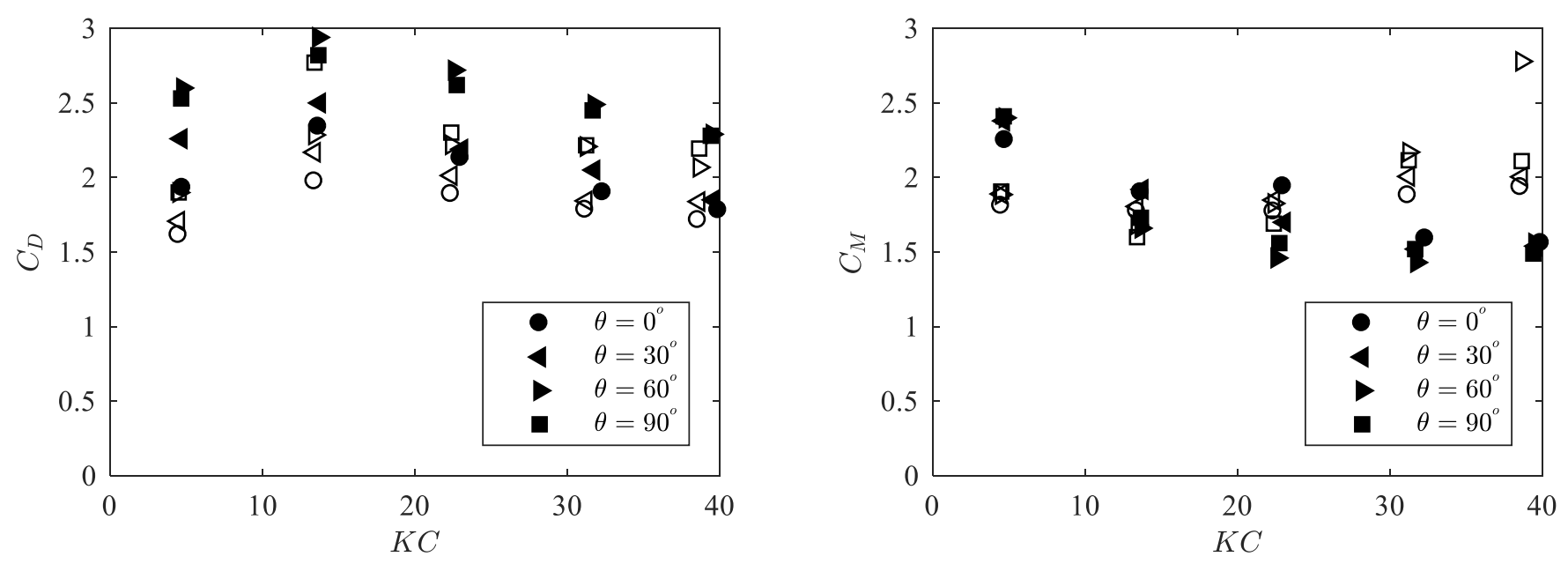

835 Figure 25 Variation of force coefficients with $K C$-number for 4 different orientations of 836 the secondary structure. Structure: Jacket leg with secondary structures. Filled

837 symbols: Physical model tests. Empty symbols: CFD test.

838

\section{Summary and conclusions}

840 In this study experimental and numerical analyses have been carried out to study

841 the effect of secondary structures, e.g. boat-landings, on a circular cylinder as part of a

842 support structure for offshore wind turbines. The study consisted of validation tests of

843 forces on a circular cylinder in oscillatory flow, which were followed with studies of

844 forces of the effect of the secondary structures on the total load. The secondary

845 structures were represented by circular cylinders placed at different orientations around

846 the main circular cylinder. All the analyses assumed uniform transverse to the flow

847 direction. Therefore the numerical simulations with OpenFOAM were carried out on

848 two spatial dimensions.

849 The validation tests with a circular cylinder without secondary structures showed

850 good agreement between the physical and numerical results, as well as to the results

851 available in the open literature. The comparison included force coefficients as well as

852 qualitative comparison of force time history. Lift force coefficients appeared larger for

853 the numerical calculations compared to the experimental results. This difference could

854 be attributed to the two-dimensional set-up of the CFD-model.

855 The validation gave confidence in both the physical and numerical methodologies

856 abilities to accurately reproduce the effect of the confined space in between the

857 structural parts, acceleration of flow, vortex shedding and coherent structures of 
turbulence around the secondary structures relative to the main cylinder on the simulated force response time series.

The orientation of the secondary structures had a clear influence on the drag related part of the force as the drag coefficient $C_{D}$ when the secondary structure was orientated towards $\theta=90^{\circ}$, i.e. perpendicular to the flow direction. When the orientation was large a large part of the structure was exposed to the flow which was found to increase the drag coefficient. The inertia part of the force, i.e. the inertia force coefficient $C_{M}$, was only affected by the orientation to small extent. The volume of the structure is important to the inertia force, and as this did not change with changing orientation, it could be expected that the inertia coefficient would be unaffected as we found.

In general there was a good agreement of the estimated force coefficients between the experimental and numerical results. It should be noted that some slight discrepancies existed for $C_{D}$ at very low $K C$-number $(K C<5)$ and for $C_{M}$ at very high $K C$-numbers $(K C>25-30)$. In both cases these discrepancies seem negligible due to the ranges of the $K C$-number being in the inertia dominated regime when $K C<5$ and in the drag dominated when $K C>20-30$.

\section{Acknowledgement}

Ørsted A/S supported the study. Their support is greatly appreciated.

\section{References}

Alam, M.M., Sakamoto, H., Zhou, Y., 2005. Determination of flow configurations and fluid forces acting on two staggered circular cylinders of equal diameter in crossflow. J. Fluids Struct. 21, 363-394. doi:10.1016/j.jfluidstructs.2005.07.009

Bearman, P.W., Downie, M.J., Graham, J.M.R., Obasaju, E.D., 1986. Force on a circular cylinder in viscous oscillatory flow at low keulegan - carpenter numbers. J. Fluid Mech. 154, 337-356. doi:10.1017/S0022112086002999

Bearman, P.W., Graham, J.M.R., Obasaju, E.D., 1984. A model equation for the transverse forces on cylinders in oscillatory flows. Appl. Ocean Res. 6, 166-172. doi:10.1016/0141-1187(84)90006-3

Bjørke, A.S., Christensen, E.D., Carstensen, S., Petersen, K.P., Mandviwalla, X., 
Kristiansen, T., Baarholm, R., 2018. Study of hydrodynamic forces on complex structures, in: Proceedings of the 37th International Conference on Ocean, Offshore and Arctic Engineering, OMAE2018. pp. 1-10.

Bredmose, H., Hansen, E.A., Pedersen, L.M., Skourup, J., Christensen, E.D., Mitzlaff, A., 2006. Numerical reproduction of extreme wave loads on a gravity wind turbine foundation, in: Proceedings of the International Conference on Offshore Mechanics and Arctic Engineering - OMAE. doi:10.1115/OMAE2006-92258

Bredmose, H., Jacobsen, N.G., 2011. Vertical Wave Impacts on Offshore Wind Turbine Inspection Platforms, in: In Proceedings of the ASME 2011 30th International Conference on Ocean, Offshore and Arctic Engineering, OMAE2011. pp. 645654. doi:10.1115/OMAE2011-49785

Bryndum, M.B., Jacobsen, V., Tsahalis, D.T., 1992. Hydrodynamic forces on pipelines: Model tests. J. Offshore Mech. Arct. Eng. 114, 231-241.

Caretto, L.S., Gosman, A.D., Patankar, S.V., Spalding, D.B., 1973. Two calculation procedures for steady, three-dimensional flows with recirculation, in: Proceedings of the Third International Conference on Numerical Methods in Fluid Mechanics, Lecture Notes in Physics, Volume 19. Springer-Verlag, Paris, pp. 60-68.

Chen, H., Christensen, E.D., 2016. Investigations on the porous resistance coefficients for fishing net structures. J. Fluids Struct. 65, 76-107. doi:10.1016/j.jfluidstructs.2016.05.005

Cheng, X., Wang, Y., Ren, B., Wang, G., 2012a. Numerical study of hydrodynamci forces on a submarine piggyback pipeline under wave action, in: Proceedings of the 31st International Conference on Ocean, Offshore and Arctic Engineering, OMAE2012. Rio de Janeiro, Brazil, pp. 177-183.

Cheng, X., Wang, Y., Ren, B., Wang, G., 2012b. Numerical Study of Hydrodynamic Forces on a Submarine Piggyback Pipeline Under Wave Action. J. Offshore Mech. Arct. Eng. 135, 021701-1 to 021701-8. doi:10.1115/OMAE2012-83214

Cheng, X., Wang, Y., Wang, G., 2012c. Hydrodynamic Forces on a Large Pipeline and a Small Pipeline in Piggyback Configuration under Wave Action. J. Waterw. Port, Coastal, Ocean Eng. 138, 394-405. doi:10.1061/(ASCE)WW.1943-5460.0000144 Christensen, E.D., Bredmose, H., Hansen, E.A., 2005. Extreme wave forces and wave run-up on offshore wind- turbine foundations, in: Copenhagen Offshore Wind. pp. 
921

922

923

924

925

926

927

928

929

930

931

932

933

934

935

936

937

938

939

940

941

942

943

944

945

946

947

948

949

950

951

952

1-10.

Christensen, E.D., Lohmann, I.P., Hansen, H.F., Haerens, P., Mercelis, P., Demuynck, A., 2011. Irregular wave loads on a gravity based foundation in shallow water, in: Proceedings of the ASME 2011 30th International Conference on Ocean, Offshore and Arctic Engineering. ASME, Rotterdam, The Netherlands, pp. 1-10.

Christensen, E.D., Stuiver, M., Guanche, R., Møhlenberg, F., Schouten, J.-J., Svenstrup Pedersen, O., He, W., Zanuttigh, B., Koundouri, P., 2015. Go offshore Combining food and energy production. Technical University of Denmark. Department of Mechanical Engineering, Kgs. Lyngby, Denmark.

Damsgaard, M.L., Gravesen, H., Andersen, T.L., 2007. Design Loads on Platforms on Offshore wind Turbine Foundations with Respect to Vertical Wave Run-up, in: In Proceedings of Offshore Wind 2007 Conference \& Exhibition The European Wind Energy Association. p. 7.

Hover, F.S., Tvedt, H., Triantafyllou, M.S., 2001. Vortex-induced vibrations of a cylinder with tripping wires. J. Fluid Mech. 448, 175-195. doi:10.1017/S0022112001005985

Igarashi, T., 1986. Effect of Tripping Wires on the flow around a Circular Cylinder Normal to an Airstream. Bull. JSME 29, 2917-2924.

Issa, R.I., 1986. Solution of the implicitly discretised fluid flow equations by operatorsplitting. J. Comput. Phys. 62, 40-65. doi:10.1016/0021-9991(86)90099-9

James, D.F., Truong, Q.-S., 1972. Wind load on Cylinder with Spanwise Protrusion. J. Eng. Mech. Div. 98, 1573-1589.

Jensen, B., Carstensen, S., Christensen, E.D., 2018. Mixing of stratified flow around bridge piers in steady current. J. Hydraul. Eng. 144, 1-13. doi:10.1061/(ASCE)HY.1943-7900.0001481

Justesen, P., 1991. A numerical study of oscillating flow around a circular cylinder. J. Fluid Mech. 222, 157-196. doi:10.1017/S0022112091001040

Justesen, P., 1989. Hydrodynamic forces on large cylinders in oscillatoty flow. J. Waterw. Port, Coastal, Ocean Eng. 115, 497-514.

Kalghatgi, S.G., Sayer, P.G., 1997. Hydrodynamic Forces on Piggyback Pipeline Configurations. J. Waterw. Port, Coastal, Ocean Eng. 123, 16-22. doi:10.1061/(ASCE)0733-950X(1997)123:1(16) 
953

954

955

956

957

958

959

960

961

962

963

964

965

966

967

968

969

970

971

972

973

974

975

976

977

978

979

980

981

982

983

984

Menter, F.R., 1994. Two-Equation Eddy-Viscosity Turbulence Models for Engineering Applications. AIAA J. 32, 1598-1605.

OpenFOAM, The open source CFD toolbox [WWW Document], 2018. URL https://www.openfoam.com/

Paulsen, B.T., Bredmose, H., Bingham, H.B., Jacobsen, N.G., 2014. Forcing of a bottom-mounted circular cylinder by steep regular water waves at finite depth. J. Fluid Mech. 755, 1-34. doi:10.1017/jfm.2014.386

Pearcey, H.H., Cash, R.F., Salter, I.J., 1982. Flow Past Circular Cylinders: Simulation of Full-Scale flows at Model Scale. NMI R 131; OT-R-8208, Feltham.

Saghafian, M., Stansby, P.K., Saidi, M.S., Apsley, D.D., 2003. Simulation of turbulent flows around a circular cylinder using nonlinear eddy-viscosity modelling: Steady and oscillatory ambient flows. J. Fluids Struct. 17, 1213-1236. doi:10.1016/S08899746(03)00066-5

Santo, H., Taylor, P.H., Day, A.H., Nixon, E., Choo, Y.S., 2018a. Current blockage and extreme forces on a jacket model in focussed wave groups with current. J. Fluids Struct. 78, 24-35. doi:10.1016/j.jfluidstructs.2017.12.015

Santo, H., Taylor, P.H., Williamson, C.H.K., Choo, Y.S., 2018b. The relative-velocity version of the Morison equation for obstacle arrays in combined steady, low and high frequency motion. J. Fluid Mech. 842, 188-214. doi:10.1017/jfm.2018.130

Sarpkaya, T., 1986. In-Line and Transverse Forces on Smooth and Rough Cylinders in Oscillatory Flow at High Reynolds Numbers. Monterey, California. doi:10.1177/003754970107600513

Sarpkaya, T., 1977. In-line and transverse forces on cylinders near a wall in oscillatory flow at high Reynolds numbers, in: Proceeding of the 9th Annual Technology Conference, Paper OTC 2898. Housto, Texas, pp. 3:161-166.

Sarpkaya, T., 1976. In-line and transverse forces on smooth and sand-roughened cylinders in oscillatory flow at high reynolds numbers, NPS-69SL76062. Monterey, California.

Sumer, B.M., Fredsøe, J., 2006. Hydrodynamics Around Cylindrical Structures (Revised Edition), Advanced Series on Ocean Engineering. World Scientific Publishing Co. Pte. Ltd., New Jersey. London, Singapore, Beijing, Shanhai, Hong Kong, Taipei, Chennai. doi:10.1142/9789812772770 
985 Wen, P., Qiu, W., 2017. Investigation of drag crisis phenomenon using CFD methods.

986 Appl. Ocean Res. 67, 306-321. doi:10.1016/j.apor.2017.07.012

987 Zhao, M., Cheng, L., Teng, B., 2007. Numerical modeling of flow and hydrodynamic

988 forces around a piggyback pipeline near the seabed. J. Waterw. Port Coast. Ocean

989 Eng. 133, 286-295. doi:Doi 10.1061/(Asce)0733-950x(2007)133:4(286)

990

991 


\section{Figure text}

993 Figure 1 Schematic description of the test flume.

994 Figure 2 The two layouts, Left: Monopile with secondary structures, Right: Jacket leg $995 \quad$ with secondary structures.

996 Figure 3 Definition sketch (schematic) of the monopile set-up with the orientation of the 997 secondary structures relative to the direction of oscillatory motion defined by $\theta$.

998 Figure 4 (a) Monopile without secondary structures (b) Monopile with secondary 999 structures.

1000 Figure 5 Left) Support frame cantilevering the monopile model. (Right) Close-up of 1001 connection between support frame and the suspended monopile model

1002 Figure 6 Sketch of arrangement of the load cells.

1003 Figure 7 Illustration of the model attached to the carriage. The measuring section was $100430 \mathrm{~cm}$ high. The total water depth was $0.75 \mathrm{~m}$. The top of the measuring section $1005 \quad$ was 0.23 melow the free water surface.

1006 Figure 8 Mesh for $6 m$ diameter monopile with secondary structures at $60^{\circ}$ rotation, $k s=0.0045 \mathrm{~m}$. a) View of full domain. b) Close-up of the monopile and secondary structures with graded refinement. c) Close-up of secondary structures with partial view of monopile with boundary layer elements visible.

1010 Figure 9 Illustration of the vortex formation in a case with $K C=20$.

1011 Figure 10 CFD-model, $K C=10.5 C F D$-model, $k_{s} / D=0.01$. Resembles result presented in (Sarpkaya, 1976), in his fig. 24i. In his case $K C=12$.

1013 Figure 11 CFD-model, $K C=15.7, k_{s} D=0.01$. Resembles result presented in

1014 (Sarpkaya, 1976), in his fig. 24h. In his case $K C=16.3$.

1015 Figure $12 \mathrm{KC}=26.2, \mathrm{k}_{s} / D=0.001$. Resembles result presented in (Sarpkaya, 1976), in 1016 his fig. 24e. In his case $K C=25.4$.

1017 Figure 13 Two examples of comparisons of force time-series from physical (measured) $1018 \quad$ and $C F D$ models. The left panel had $K C_{\text {mea }}=7.7$, and $K C_{C F D}=7.9$. The right panel 1019 had $K C_{m e a}=31.8$, and $K C_{C F D}=31.3$. The relative roughness $w a s$ in the order, $1020 \quad k_{s} / D \square 1.25 \cdot 10^{-3}$.

1021 Figure 14 The variation of $C_{D}$ and $C_{M}$ over the tested range of the KC-number for the 1022 monopile and jacket leg model without secondary structures attached. The relative 
roughness was in the order, $k_{s} / D \square 1.25 \cdot 10^{-3}$ except for the case of (Justesen, 1989), where it was $k_{s} / D \square 3 \cdot 10^{-3}$.

1025 Figure 15 The Maximum lift force coefficient, $C_{L, \max }$, for over the tested range of the $K C$-number for the monopile and jacket leg model without secondary structures attached. The relative roughness was in the order, $k_{s} / D \square 1.25 \cdot 10^{-3}$.

1028 Figure 16 Time series of inline forces for $K C=5.2, k_{s} / D=1.25 * 10^{-3}$. Mono-pile with secondary structures at $0^{\circ}$ orientation.

1030 Figure 17 Time series of inline forces for $K C$-numbers: $K C_{C F D}=13.1, K C_{\text {Phys. }}=12.9$, $k_{s} / D=1.25 \cdot 10^{-3}$. Mono-pile with secondary structures at $0^{\circ}$ orientation.

1032 Figure 18 Time series of inline forces for $K C$-numbers: $K C_{C F D}=31.3, K C_{P h y s}=32.3$, $k_{s} / D=1.25 \cdot 10^{-3}$. Jacket-leg with secondary structures at $0^{\circ}$ orientation.

Figure 19 Time series of inline forces for $K C$-numbers: $K C_{C F D}=31.3, K C_{P h y s}=32.3$, $. . k_{s} / D=1.25 \cdot 10^{-3}$. Jacket-leg with secondary structures $90^{\circ}$ orientation.

1036 Figure $20 \mathrm{KC}=5$ 5.2. Monopile with secondary structures

1037 Figure $21 \mathrm{KC}=10$, Monopile with secondary structures

1038 Figure $22 \mathrm{KC}=13$, Monopile with secondary structures

1039 Figure $23 \mathrm{KC}=31$, Jacket leg with secondary structure

1040 Figure 24 Variation of force coefficients with KC-number for 4 different orientations of

1041 the secondary structure. Structure: Monopile with secondary structures. Filled

1042 symbols: Physical model tests. Empty symbols: CFD test.

1043 Figure 25 Variation of force coefficients with KC-number for 4 different orientations of 1044 the secondary structure. Structure: Jacket leg with secondary structures. Filled $1045 \quad$ symbols: Physical model tests. Empty symbols: CFD test. 